\title{
Stability and Persistence of an Avian Influenza Epidemic Model with Impacts of Climate Change
}

\author{
Xiao-Yan Zhao, ${ }^{1}$ Shu-Min Guo, ${ }^{2}$ Mini Ghosh, ${ }^{3}$ and Xue-Zhi Li ${ }^{4}$ \\ ${ }^{1}$ Department of Basic Courses, Henan Quality Engineering Vocational College, Pingdingshan 467001, China \\ ${ }^{2}$ College of Mathematics and Statistics, Shaoguan University, Shaoguan 512005, China \\ ${ }^{3}$ School of Advanced Sciences, VIT University, Chennai Campus, Chennai 600127, India \\ ${ }^{4}$ College of Mathematics and Physics, Anyang Institute of Technology, Anyang 455000, China
}

Correspondence should be addressed to Xue-Zhi Li; xzli66@126.com

Received 14 December 2015; Accepted 11 April 2016

Academic Editor: Ciprian G. Gal

Copyright (C) 2016 Xiao-Yan Zhao et al. This is an open access article distributed under the Creative Commons Attribution License, which permits unrestricted use, distribution, and reproduction in any medium, provided the original work is properly cited.

The growing number of reported avian influenza cases has prompted awareness of the importance of research methods to control the spread of the disease. Seasonal variation is one of the important factors that affect the spread of avian influenza. This paper presents a "nonautonomous" model to analyze the transmission dynamics of avian influenza with the effects of climate change. We obtain and discuss the global stability conditions of the disease-free equilibrium; the threshold conditions for persistence, permanence, and extinction of the disease; and the parameters with periodicity for controlling and eliminating the avian influenza.

\section{Introduction}

The avian influenza (avian flu or bird flu) refers to the influenza that is caused by viruses adapted to birds. The influenza A virus, only species of influenza virus, can cause influenza in birds and some mammals. The strains of all subtypes of influenza A virus have been isolated from wild birds and at the current understanding some isolates of influenza A virus can cause severe disease both in domestic poultry and in humans though rarely [1] since there is always a possibility of the transmission of viruses from wild aquatic birds to domestic poultry and through them it can cause an outbreak or give rise to human influenza pandemics.

A human was infected with avian influenza from birds in Hong Kong in 1997 and earlier in Hong Kong flu of 1968. Since then the infection to human from the avian influenza has occurred successively. The WHO has warned of a substantial risk of pandemic avian influenza in the near future. The outbreak of avian influenza is related to the changes in climate and usually it happens in spring and winter. The influenza A viruses are highly sensitive to temperature and with the increase of ambient temperature the survival time of the virus is shortened since in summer and autumn the sunlight is stronger and the sun's ultraviolet rays are able to kill the virus. However, the same is not true in winter and rainy weather and these weather conditions offer the virus an opportunity to thrive. In this regard, it is important to analyze the impact of the weather seasons on spread of the avian influenza. There exist many diseases that show seasonal behavior, for example, allergic rhinitis, pityriasis rosea, coronary heart disease, psoriasis, and avian influenza. These infections are also periodic and the transmission rates and other parameters can vary because of the force of season [2-10].

Mathematical modelling of avian influenza is not new. In recent years, Iwami et al. [11-14] formulated and analyzed several mathematical models for avian flu for better understanding of transmission and control of this disease. In 2007, Iwami et al. [11] studied a simple mathematical model for avian influenza by considering both human and bird populations. Their findings suggested that culling of infected bird will not be enough to control the avian influenza when mutant avian influenza has already occurred. The human infected with mutant avian influenza must be quarantined to control the further occurrence of this disease. In 2008, Iwami et al. [12] further extended their previous model and focused on the effectivity of the two prevention policies, namely, the 
elimination policy and the quarantine policy, and it is found that the effectivity of the policies depends on the rate of transmission, rate of mutation, and the virulence evolution. Later, in 2009 [13], they investigated the relations between evolution of the virulence and effectiveness of pandemic control measures after the emergence of mutant avian influenza. Also, they presented a deterministic path-structured model in heterogeneous areas in the same year [14]. Kwon et al. [15] discussed the immune responses and pathogenesis in immune compromised chickens in response to the infection with the H9N2 low pathogenic avian influenza virus. Wang et al. [16] studied the person to person transmission of H5N1 in China. Some additional results have also been obtained in [17-19]. But in all of the above discussed models impact of climate is not taken into account and models were based on system of autonomous differential equations. In the present work, we have framed a mathematical model incorporating seasonal variations in the parameters which makes this system nonautonomous but it is more realistic. It can be noted here that some of our results are comparable with the results of [3] because our approach is similar to them. However, our contribution is that our presented model is more complicated as compared to [3] and we consider avian influenza model involving both human world and bird world. Also, in the human world we have class of humans infected with mutant avian influenza in addition to usual infectious class. We have analyzed the system for the permanence and extinction of the infectives in both populations.

The rest of this paper is organized as follows: Section 2 presents the model; Section 3 discusses the global stability conditions of the disease-free equilibrium in the absence of human infected with the mutant avian influenza; Section 4 presents the global stability of disease-free equilibrium in the presence of human infected with the mutant avian influenza; Section 5 deals with the persistence, permanence, and extinction analysis of the bird world and human world; and finally Section 6 concludes the paper and summarizes our major results and contributions.

\section{The Model}

In this section, we are presenting a model to interpret the spread of avian influenza between the bird world and the human world. The epidemic model considered here is as follows:

$$
\begin{aligned}
\frac{d x(t)}{d t}= & \lambda(t)-b(t) x(t)-\beta_{1}(t) x(t) y(t), \\
\frac{d y(t)}{d t}= & \beta_{1}(t) x(t) y(t)-(b(t)+m(t)) y(t), \\
\frac{d S(t)}{d t}= & \Lambda(t)-\mu(t) S(t) \\
& -\left(\beta_{2}(t) y(t)+\beta_{3}(t) H(t)\right) S(t), \\
\frac{d I(t)}{d t}= & \beta_{2}(t) S(t) y(t)-(\mu(t)+d(t)+\varepsilon(t)) I(t),
\end{aligned}
$$

$$
\begin{aligned}
\frac{d H(t)}{d t}= & \beta_{3}(t) S(t) H(t)+\varepsilon(t) I(t) \\
& -(\mu(t)+\alpha(t)+\gamma(t)) H(t), \\
\frac{d R(t)}{d t}= & \gamma(t) H(t)-\mu(t) R(t) .
\end{aligned}
$$

System (1) is divided into the bird world and the human world. In the bird world, avian influenza-free birds and the infectious birds at time $t$ are denoted by $x(t)$ and $y(t)$, respectively. Here $\lambda(t)$ is the recruitment rate of the birds; $b(t)$ and $m(t)$ represent the natural death rate and the disease-related death rate, respectively. The incidence of newly infected birds is given by $\beta_{1}(t) x(t) y(t)$. The human world can be divided into four classes: susceptible $S(t)$, infectious $I(t)$, human infected with mutant avian influenza $H(t)$, and recovered human $R(t)$ who is recovered from mutant avian influenza, respectively. Here $\Lambda(t)$ is the rate of recruitment into the human world and it is assumed that all newly recruited humans are susceptible. $\mu(t)$ is the natural death rate; $d(t)$ and $\alpha(t)$ are the disease-related death rates in $I(t)$ and $H(t)$ classes, respectively; $\gamma(t)$ is the rate of recovery in $H(t)$ class; $\varepsilon(t)$ is the mutation rate from $I(t)$ to $H(t)$ class at time $t ; \beta_{2}(t) S(t) y(t)$ and $\beta_{3}(t) S(t) H(t)$ represent the force of infection at time $t$ by humans infected with avian influenza and mutant avian influenza, respectively.

In this paper, we consider the infection rate to be time dependent; for example, infection rates $\beta_{1}(t), \beta_{2}(t), \beta_{3}(t)$ and other parameters vary with the time, and the periodicities are the same due to the effect of social and other factors. We assume that $\beta_{1}(t), \beta_{2}(t), \beta_{3}(t)$ are the periodic function of the same period and are given in the form of a sinusoidal function as follows:

$$
\beta_{i}(t)=k_{1}+k_{2} \cos (2 \pi t-\varphi) \quad(i=1,2,3) .
$$

In fact this kind of periodic function is considered by several other researchers in different context. Li and Qi [20] formulated the peripheral blood in the following form: $T(t)=$ $K_{0}+K_{1} \cos (2 \pi t-\varphi)$. Also in [3], authors define the periodic function $p(t)=p_{0}(1+\varepsilon \cos (w t-\phi))$ with $\varepsilon \ll 1$ and $p$ is the constant vector population $p_{0}$, but with $p$ replaced by the average vector population.

First we analyze the model system (1) by assuming

$$
\begin{aligned}
& \lambda(t)=\lambda, \\
& b(t)=b, \\
& m(t)=m, \\
& \Lambda(t)=\Lambda, \\
& \mu(t)=\mu, \\
& \varepsilon(t)=\varepsilon, \\
& d(t)=d, \\
& \alpha(t)=\alpha, \\
& \gamma(t)=\gamma .
\end{aligned}
$$


Hence the model system (1) now reduces to the following system:

$$
\begin{aligned}
\frac{d x}{d t} & =\lambda-b x(t)-\beta_{1}(t) x(t) y(t), \\
\frac{d y}{d t} & =\beta_{1}(t) x(t) y(t)-(b+m) y(t), \\
\frac{d S}{d t} & =\Lambda-\mu S(t)-\left(\beta_{2}(t) y(t)+\beta_{3}(t) H(t)\right) S(t), \\
\frac{d I}{d t} & =\beta_{2}(t) S(t) y(t)-(\mu+d+\varepsilon) I(t), \\
\frac{d H}{d t} & =\beta_{3}(t) S(t) H(t)+\varepsilon I(t)-(\mu+\alpha+\gamma) H(t), \\
\frac{d R}{d t} & =\gamma H(t)-\mu R(t), \\
x(0) & >0, y(0) \geq 0, S(0)>0, I(0) \geq 0, H(0) \geq 0, R(0) \geq 0 .
\end{aligned}
$$

By straightforward calculation, it is easy to see that system (4) has the disease-free equilibrium $E_{0}(\lambda / b, 0, \Lambda / \mu, 0,0,0)$. Let

$$
r_{0}=\frac{\overline{\beta_{1}} \lambda}{b(b+m)}
$$

where $\overline{\beta_{1}}=(1 / w) \int_{0}^{w} \beta_{1}(t) d t$ denotes the average value of $\beta_{1}(t)$. We denote

$$
\begin{aligned}
& r_{0}^{\max }=\frac{\beta_{1} \lambda}{b(b+m)} \\
& r_{0}^{\min }=\frac{\beta_{1}^{\prime} \lambda}{b(b+m)}
\end{aligned}
$$

where

$$
\begin{aligned}
& \beta_{1}=\sup _{0 \leq t \leq w} \beta_{1}(t), \\
& \beta_{1}^{\prime}=\min _{0 \leq t \leq w} \beta_{1}(t) .
\end{aligned}
$$

\section{The Global Asymptotic Stability and the Persistence of System (4) with $(H=0)$}

Theorem 1. All solutions of system (4) are positive and ultimately bounded; that is, there exists a positive constant $M>0$, such that all solutions of system (4) satisfy $x(t)<M$, $y(t)<M, S(t)<M, I(t)<M, H(t)<M$, and $R(t)<M$ for all large $t$.

Proof. We note that

$$
N(t)=n_{1}(t)+n_{2}(t)
$$

where $n_{1}(t)=x(t)+y(t)$ and $n_{2}(t)=S(t)+I(t)+H(t)+R(t)$.

We consider the bird world first. Since $y(t)=0$ is a constant solution of system (4), by the uniqueness and continuity of the solutions with respect to the initial conditions, we have $y(t)>0$ for all $t>0$.

Next, we will prove that $x(t)$ is nonnegative. Suppose that $x(t)$ is not always positive. Let $\tau_{1}>0$ be the first time such that $x\left(\tau_{1}\right)=0$. By the first equation of system (4), we have $x^{\prime}\left(\tau_{1}\right)=\lambda>0$, which means $x(t)<0$ for $t \in\left(\tau_{1}-\epsilon, \tau_{1}\right)$, where $\epsilon$ is an arbitrarily small positive constant. It leads to a contradiction. Thus $x(t)$ is always positive. Let $v_{1}(t)=x(t)+$ $y(t)$. Since all solutions of (4) are positive, we have $v_{1}^{\prime}(t)=$ $\lambda-b x(t)-b y(t)-m y(t) \leq \lambda-b v_{1}(t)$. Therefore, $v_{1} \leq \lambda / b+1$ for $t>t_{1}$. Since $x(t) \leq v_{1}, y(t) \leq v_{1}, x(t)$ and $y(t)$ are ultimately bounded. There exists a positive constant $M_{1}>\lambda / b+1$ such that $x(t)<M$ and $y(t)<M$.

Similarly, in the human world, $H=0$ is a constant solution of system (4). By the uniqueness and continuity of the solutions with respect to the initial conditions, we get $H(t)>0$ for any $t>0$. Similarly, suppose that $S(t)$ is not always positive. Let $\tau_{2}>0$ be the first time such that $S\left(\tau_{2}\right)=0$. Then from the third equation of system $(4), S^{\prime}\left(\tau_{2}\right)=$ $\Lambda-\mu S\left(\tau_{2}\right)-\left(\beta_{2}\left(\tau_{2}\right) y\left(\tau_{2}\right)+\beta_{3}\left(\tau_{2}\right) H\left(\tau_{2}\right)\right) S\left(\tau_{2}\right)=\Lambda>0$ which means $S(t)<0$ for $t \in\left(\tau_{2}-\eta, \tau_{2}\right) ; \eta$ is an arbitrarily small positive constant. It leads to a contradiction. Thus $S(t)$ is always positive. In the same way, we can show that $I(t)$ and $R(t)$ are always positive. Let $v_{2}(t)=S(t)+I(t)+H(t)+R(t)$. Then we have $v_{2}^{\prime}(t)=\Lambda-\mu v_{2}(t)-d I(t)-\alpha H(t) \leq \Lambda-\mu v_{2}(t)$. Therefore, $v_{2}(t) \leq \Lambda / \mu+1$ for $t>t_{2}$. Thus the human system is ultimately bounded for some positive constant $M_{2}$. Let $t_{0}=\max \left\{t_{1}, t_{2}\right\}$. The bird-human system (4) is bounded by $M$ for $t \geq t_{0}$. This completes the proof of Theorem 1 .

Theorem 2. Let

$$
\begin{aligned}
& x^{\infty}=\limsup _{t \rightarrow \infty} x(t), \\
& S^{\infty}=\limsup _{t \rightarrow \infty} S(t) .
\end{aligned}
$$

Then $x^{\infty} \leq \bar{x}, S^{\infty} \leq \bar{S}$.

Proof. By the first equality of system (4), we have

$$
x^{\prime}(t)=\lambda-b x(t)-\beta_{1}(t) x(t) y(t) \leq \lambda-b x(t) .
$$

We integrate the inequality over $[0, t]$ :

$$
\int_{0}^{t} x^{\prime}(s) d s \leq \int_{0}^{t}(\lambda-b x(s)) d s .
$$

Thus, we have

$$
x(t) \leq \bar{x}+|x(0)-\bar{x}| e^{-b t} .
$$

We choose $\epsilon_{0}>0$, which is arbitrarily small, and $t_{3}$ is large enough, such that $|x(0)-\bar{x}| e^{-b t} \leq \epsilon_{0}$. Hence $x(t) \leq \bar{x}+\epsilon_{0}$ for $t \geq t_{3}$. Thus, for $T \geq t_{3}, \sup _{t \geq T} x(t) \leq \bar{x}+\epsilon_{0}$. Let $T \rightarrow \infty$, and thus we have $x^{\infty} \leq \bar{x}+\epsilon_{0}$, and hence $x^{\infty} \leq \bar{x}$. We can proof $S^{\infty}<\bar{S}$ by using the similar method given in the proof of $x^{\infty} \leq \bar{x}$. This completes the proof of Theorem 2 . 
We will prove the global stability results of model (4) for $H=0$. When $H=0$, system (4) reduces to a new system:

$$
\begin{aligned}
& \frac{d x}{d t}=\lambda-b x(t)-\beta_{1}(t) x(t) y(t), \\
& \frac{d y}{d t}=\beta_{1}(t) x(t) y(t)-(b+m) y(t), \\
& \frac{d S}{d t}=\Lambda-\mu S(t)-\beta_{2}(t) S(t) y(t), \\
& \frac{d I}{d t}=\beta_{2}(t) S(t) y(t)-(\mu+d) I(t), \\
& x(0)>0, y(0) \geq 0, S(0)>0, I(0) \geq 0 .
\end{aligned}
$$

We have the disease-free equilibrium $E_{0}(\lambda / b, 0)$ (bird world) and $\bar{E}_{0}(\lambda / b, 0, \Lambda / \mu, 0)$ (the bird-human world).

Theorem 3. The disease-free equilibria $E_{0}$ and $\bar{E}_{0}$ are globally asymptotically stable if $r_{0}^{\max }<1$.

Proof. By the second equality of system (4), we have

$$
\frac{y^{\prime}(t)}{y(t)}=\beta_{1}(t) x(t)-(b+m) \text {. }
$$

Integrating the above differential equation over $\left[t_{0}, t_{0}+t\right]$, we have

$$
\frac{y\left(t_{0}+t\right)}{y\left(t_{0}\right)} \leq e^{\int_{t_{0}}^{t_{0}+t}\left[\beta_{1}(s) x(s)-(b+m)\right] d s} .
$$

By Theorem 2, we know that given $\epsilon_{0}>0$ there exists $t_{0}$ such that $x(t) \leq \bar{x}+\epsilon_{0}$ for all $t \geq t_{0}$. Hence

$$
\begin{aligned}
y\left(t_{0}+t\right) & \leq y\left(t_{0}\right) e^{\int_{t_{0}}^{t_{0}+t}\left[\beta_{1}(s)\left(\bar{x}+\epsilon_{0}\right)-(b+m)\right] d s} \\
& \leq y\left(t_{0}\right) e^{\int_{t_{0}}^{t_{0}+t}\left[(b+m)\left(\beta_{1}(s) \bar{x} /(b+m)-1\right)+\beta_{1}(s) \epsilon_{0}\right] d s} \\
& \leq y\left(t_{0}\right) e^{\int_{t_{0}}^{t_{0}+t}\left[(b+m)\left(r_{0}^{\max }-1\right)+\beta_{1}(s) \epsilon_{0}\right] d s} .
\end{aligned}
$$

We have $(b+m)\left(r_{0}^{\max }-1\right)+\beta_{1}(t) \epsilon_{0} \rightarrow(b+m)\left(r_{0}^{\max }-1\right)<0$ if $r_{0}^{\max }<1$ and $\epsilon_{0} \rightarrow 0$; supposing $\epsilon_{0}$ is small enough, we assume that $K \equiv(b+m)\left(1-r_{0}^{\max }\right)-\beta_{1}(t) \epsilon_{0}>0$. From (16), we obtain

$$
y\left(t_{0}+t\right) \leq y\left(t_{0}\right) e^{-k t}
$$

Hence $y\left(t_{0}+t\right) \rightarrow 0$ as $t \rightarrow \infty$. We suppose that $\epsilon_{1}>0$ is arbitrarily small. There exists $t_{1}$ such that $y(t) \leq \epsilon_{1}$ for all $t \geq t_{1}$.

Since $y(t) \rightarrow 0$ as $t \rightarrow \infty$, there exists $t_{2}$ such that $y(t) \leq$ $\epsilon_{1} / \beta_{1} \bar{x}$ for all $t \geq t_{2}$, where $\epsilon_{1}>0$ is arbitrarily small. For $t \geq t_{2}$, by the first equality of system (13), we have

$$
\dot{x}(t)=\lambda-b x-\beta_{1} x y>\lambda-\epsilon_{1}-b x .
$$

Similarly, we have $x(t) \geq\left(\lambda-\epsilon_{1}\right) / b$ for $t>t_{3}$, where $t_{3}>t_{2}$. Hence by Theorem 2, $x(t) \rightarrow \bar{x}$ as $t \rightarrow \infty$ and $\epsilon_{1}$ is arbitrarily small. Since $y(t) \rightarrow 0$ as $t \rightarrow \infty$, we assume that there exists $t_{4}>0$, such that $y(t)<\epsilon_{1} / \beta_{2} \bar{S}$ as $t>t_{4}$.

From the third equality of system (13) we have

$$
\dot{S}=\Lambda-\mu S-\beta_{2} S y \geq \Lambda-\epsilon_{1}-\mu S .
$$

By comparison theorem, we obtain

$$
\dot{S} \geq \frac{\Lambda-\epsilon_{1}}{\mu}
$$

as $t>t_{4}$. By Theorem 2, we have $S \rightarrow \bar{S}$ as $t>t_{4}$. Similarly,

$$
\dot{I}=\beta_{2}(t) S y-(\mu+d) I \leq \beta_{2}\left(\bar{S}+\epsilon_{0}\right) y-(\mu+d) I .
$$

We assume $y(t)<\epsilon_{1} / \beta_{2} \bar{S}$ as $t>t_{5}$. Hence

$$
\begin{aligned}
\dot{I} & \leq \beta_{2}\left(\bar{S}+\epsilon_{0}\right) \frac{\epsilon_{1} \epsilon_{0}}{\bar{S}}-(\mu+d) I \\
& =\varepsilon_{1}+\frac{\epsilon_{1} \epsilon_{0}}{\bar{S}}-(\mu+d) I .
\end{aligned}
$$

Thus $\dot{I} \rightarrow-(\mu+d) I$ as $\epsilon_{1}$ is arbitrarily small. Hence

$$
I(t) \leq e^{-(\mu+d) t} .
$$

When $I(t) \rightarrow 0, t \rightarrow \infty$. This completes the proof of Theorem 3.

Theorem 4. If $r_{0}^{\min }>1$, system (13) is uniformly persistent; thus there exists $\xi>0$ such that

$$
\begin{aligned}
& \liminf _{t \rightarrow+\infty} x(t) \geq \xi, \\
& \liminf _{t \rightarrow+\infty} y(t) \geq \xi, \\
& \liminf _{t \rightarrow+\infty} S(t) \geq \xi, \\
& \liminf _{t \rightarrow+\infty} I(t) \geq \xi .
\end{aligned}
$$

Proof. By the variation of the constants method for nonhomogeneous linear ordinary differential equations, the solution to the first equality of system (13) has the form

$$
x(t)=x(0) e^{-\int_{0}^{t}\left(b+\beta_{1}(s) y(s)\right) d s}+\frac{\int_{0}^{t} c e^{\int_{0}^{t}\left[b+\beta_{1}(s) y(s)\right] d s}}{e^{\int_{0}^{t}\left[b+\beta_{1}(s) y(s)\right] d s}} .
$$

Thus,

$$
\left|x(t)-\frac{\lambda}{b+\beta_{1}(t) y(t)}\right| \longrightarrow 0
$$

as $t \rightarrow \infty$. There exists $t_{1}>0$, such that

$$
\begin{aligned}
\dot{y}(t) & =\beta_{1}(t) x(t) y(t)-(b+m) y(t) \\
\geq & {\left[\beta_{1}(t)\left(\frac{\lambda}{b+\beta_{1}(t) y(t)}-\epsilon_{1}\right)-(b+m)\right] y(t) }
\end{aligned}
$$

for all $t>t_{1}$. 
Choosing $\epsilon_{1}$ which is arbitrarily small and $y(t)<M$ in Theorem 1, inequality (27) can be written as

$$
\begin{aligned}
\dot{y}(t) & >\frac{\left[\beta_{1}(t) \lambda-\left(b+\beta_{1}(t) y(t)\right)(b+m)\right] y(t)}{b+\beta_{1}(t) M} \\
& >\frac{\beta_{1}^{\prime} \lambda-b(b+m)-\beta_{1}(b+m) y(t)}{b+\beta_{1}(t) M} y(t) .
\end{aligned}
$$

Now, we consider the following differential equation:

$$
\dot{z}=\frac{\beta_{1}^{\prime} \lambda-b(b+m)-\beta_{1}(b+m) z}{b+\beta_{1}(t) M} z
$$

with $z(0)>0$, when

$$
r_{0}^{\min }=\frac{\beta_{1}^{\prime} \lambda}{b(b+m)}>1
$$

that is,

$$
\beta_{1}^{\prime} \lambda-b(b+m)=\rho \quad(\text { say })>0 .
$$

It is easy to see that

$$
\bar{z}=\frac{\rho}{\beta_{1}(b+m)}>0
$$

By standard comparison theorem, we have

$$
\liminf _{t \rightarrow \infty} y(t) \geq \bar{z}>0
$$

Hence, we have

$$
\dot{x}=\lambda-b x-\beta_{1}(t) x y \geq \lambda-\left(b+\beta_{1} M\right) x
$$

as $t$ is large enough. Thus

$$
\liminf _{t \rightarrow \infty} x(t) \geq \frac{\lambda}{b+\beta_{1} M}
$$

By the third equality of system (13), we have

$$
\dot{S}=\Lambda-\left(\mu+\beta_{2} y\right) S \geq \Lambda-\left(\mu+\beta_{2} M\right) S .
$$

By the comparison theorem we have

$$
\liminf _{t \rightarrow \infty} S(t) \geq \frac{\Lambda}{\mu+\beta_{2} M}
$$

Similarly,

$$
\dot{I}=\beta_{2}(t) S y-(\mu+d) I \geq \frac{\beta_{2}^{\prime} \Lambda M}{\mu+\beta_{2} M}-(\mu+d) I .
$$

Hence

$$
\liminf _{t \rightarrow \infty} I(t) \geq \frac{\beta_{2}^{\prime} \Lambda M}{\left(\mu+\beta_{2} M\right)(\mu+d)}
$$

Let

$$
\begin{aligned}
\xi= & \min \left\{\frac{\lambda}{b+\beta_{1} M}, \frac{\rho}{\beta_{1}(b+m)}, \frac{\Lambda}{\mu+\beta_{2} M},\right. \\
& \left.\frac{\beta_{2}^{\prime} \Lambda M}{\left(\mu+\beta_{2} M\right)(\mu+d)}\right\} .
\end{aligned}
$$

Thus, we have

$$
\begin{aligned}
& \liminf _{t \rightarrow \infty} x(t) \geq \xi, \\
& \liminf _{t \rightarrow \infty} y(t) \geq \xi, \\
& \liminf _{t \rightarrow \infty} S(t) \geq \xi, \\
& \liminf _{t \rightarrow \infty} I(t) \geq \xi .
\end{aligned}
$$

Theorem 5. The equilibrium point $E_{0}$ is unstable if $r_{0}^{\max }>r_{0}>$ 1.

Proof. It is easy to be seen from the proof of Theorem 3.

\section{Analysis of System (4) When $H \neq 0$ and $\varepsilon=0$ (Single Mutation Case)}

Under this condition, system (4) reduces to

$$
\begin{aligned}
& \frac{d x(t)}{d t}=\lambda-b x(t)-\beta_{1}(t) x(t) y(t), \\
& \frac{d y(t)}{d t}=\beta_{1}(t) x(t) y(t)-(b+m) y(t), \\
& \frac{d S(t)}{d t}=\Lambda-\mu S(t)-\left(\beta_{2}(t) y(t)+\beta_{3}(t) H(t)\right) S(t), \\
& \frac{d I(t)}{d t}=\beta_{2}(t) y(t) S(t)-(\mu+d) I(t), \\
& \frac{d H(t)}{d t}=\beta_{3}(t) S(t) H(t)-(\mu+\alpha+\gamma) H(t), \\
& \frac{d R(t)}{d t}=\gamma H(t)-\mu R(t), \\
& x(0)>0, y(0) \geq 0, S(0)>0, I(0) \geq 0, H(0) \geq 0, R(0) \geq 0 .
\end{aligned}
$$

Let

$$
\begin{aligned}
R_{0} & =\max \left\{\frac{\overline{\beta_{1}} \lambda}{b(b+m)}, \frac{\overline{\beta_{3}} \Lambda}{\mu(\mu+\alpha+\gamma)}\right\}, \\
R_{0}^{\max } & =\max \left\{\frac{\beta_{1} \lambda}{b(b+m)}, \frac{\beta_{3} \Lambda}{\mu(\mu+\alpha+\gamma)}\right\},
\end{aligned}
$$

where $\beta_{1}=\sup _{0 \leq t \leq w} \beta_{1}(t)$ and $\beta_{3}=\sup _{0 \leq t \leq w} \beta_{3}(t)$.

Theorem 6. All solutions of system (42) with initial values $x(0)>0, y(0) \geq 0, S(0)>0, I(0) \geq 0, H(0) \geq 0$, and $R(0) \geq 0$ are positive for all $t>0$ and they are all ultimately bounded. 
Proof. The theorem can be easily proved by using the similar method given in the proof of Theorem 1 . So we omit the proof of Theorem 6.

Theorem 7. The disease-free equilibrium $E_{0}^{\prime}(\lambda / b, 0, \Lambda / \mu, 0,0$, $0)$ of system (42) is globally asymptotically stable if $R_{0}^{\max }<1$.

Proof. Here we proceed in the same manner as given in the proof of Theorem 3. From Theorem 3 we have $y(t) \leq \epsilon_{1}$ for all $t \geq t_{1} \cdot \epsilon_{1}$ and $t_{1}$ are as stated in Theorem 3 and $x(t) \rightarrow \bar{x}$ as $t \rightarrow \infty$.

From the fifth equation of system (42), we have

$$
\begin{aligned}
\dot{H}= & \beta_{3}(t) S(t) H(t)-(\mu+\alpha+\gamma) H(t) \\
\leq & {\left[\beta_{3}(t)\left(\bar{S}+\epsilon_{0}\right)-(\mu+\alpha+\gamma)\right] H(t) } \\
= & (\mu+\alpha+\gamma)\left(\frac{\beta_{3}(t) \bar{S}}{\mu+\alpha+\gamma}-1\right) H(t) \\
& +\beta_{3}(t) H(t) \epsilon_{0} \\
\leq & (\mu+\alpha+\gamma)\left(\frac{\beta_{3}(t) \Lambda}{\mu(\mu+\alpha+\gamma)}-1\right) H(t) \\
& +\beta_{3}(t) H(t) \epsilon_{0} \\
\leq & (\mu+\alpha+\gamma)\left(R_{0}^{\max }-1\right)+\beta_{3}(t) H(t) \epsilon_{0} .
\end{aligned}
$$

Integrating this inequality from $t_{1}$ to $t_{1}+t$, we get

$$
\begin{aligned}
& H\left(t_{1}+t\right) \leq H\left(t_{1}\right) \\
& \quad \cdot \exp \int_{t_{1}}^{t_{1}+t}\left[(\mu+\alpha+\gamma)\left(R_{0}^{\max }-1\right)+\beta_{3}(s) \epsilon_{0}\right] d s .
\end{aligned}
$$

Since $(\mu+\alpha+\gamma)\left(R_{0}^{\max }-1\right)+\beta_{3}(s) \epsilon_{0} \rightarrow(\mu+\alpha+\gamma)\left(R_{0}^{\max }-1\right)<0$, $R_{0}^{\max }<1$ and $\epsilon_{0} \rightarrow 0$. Let $k^{\prime} \equiv(\mu+\alpha+\gamma)\left(1-R_{0}^{\max }\right)-\beta_{3}(t) \epsilon_{0}>$ 0 . From (45), we have

$$
H\left(t_{1}+t\right) \leq H\left(t_{1}\right) e^{-k^{\prime} t}
$$

Hence $H(t) \rightarrow 0$ as $t \rightarrow \infty$. So there exists $t_{2}$, such that $H(t) \leq \epsilon$ for all $t \geq t_{2}$. We supposed that $y(t) \leq \epsilon_{1} / 2 \beta_{2} \bar{S}$ as $t \geq t_{4}$. Consider

$$
\begin{aligned}
\dot{S} & =\Lambda-\mu S-\left(\beta_{2}(t) y+\beta_{3} H\right) S \\
& \geq \Lambda-\mu S-\frac{\beta_{2}(t) S \epsilon_{1}}{2 \beta_{2} \bar{S}}-\frac{\beta_{3} S \epsilon_{1}}{2 \beta_{2} \bar{S}} \geq \Lambda-\epsilon_{1}-\mu S .
\end{aligned}
$$

By comparison theorem, we have $S(t) \geq(\Lambda-\varepsilon) / \mu$ as $t \geq$ $\max \left\{t_{3}, t_{4}\right\}$. By Theorem 2 , we have $S \rightarrow \bar{S}$ as $t \geq \max \left\{t_{3}, t_{4}\right\}$.

Similarly, we have

$$
\begin{aligned}
\dot{I} & =\beta_{2}(t) S(t) y(t)-(\mu+d) I(t) \\
& \leq \beta_{2}(t)\left(\bar{S}+\epsilon_{0}\right) \frac{\epsilon_{1}}{\beta_{2} \bar{S}}-(\mu+d) I(t)
\end{aligned}
$$

as $t \geq t_{5}$.
Hence $\dot{I} \rightarrow-(\mu+d) I$. Thus

$$
I(t) \leq I(0) e^{-(\mu+d) t}
$$

as $t \geq t_{5}$. Thus $I(t) \rightarrow 0$ as $t \rightarrow \infty$. We assume that $H(t) \leq$ $\epsilon_{2} / \gamma$ as $t \geq t_{6}$. Hence $\dot{R}(t)=\gamma H(t)-\mu R(t) \leq \epsilon_{2}-\mu R(t)$. Consider $\dot{R}(t) \rightarrow-\mu R(t)$ as $\epsilon_{2}$ is small enough. Thus

$$
R(t) \leq R(0) e^{-\mu t}
$$

Consider $R(t) \rightarrow 0$ as $t \rightarrow \infty$. This completes the proof of Theorem 7.

\section{The Permanence and Extinction of the Bird World and Human World}

In this section we prove the permanence and extinction results of bird world and human world by extending earlier results of [3] where the authors dealt with the simplified system.

We consider the following bird and human systems which are the subsystems of system (1):

$$
\begin{aligned}
\frac{d x(t)}{d t}= & \lambda(t)-b(t) x(t)-\beta_{1}(t) x(t) y(t), \\
\frac{d y(t)}{d t}= & \beta_{1}(t) x(t) y(t)-(b(t)+m(t)) y(t), \\
\frac{d S(t)}{d t}= & \Lambda(t)-\mu(t) S(t) \quad y(0) \geq 0 . \\
& -\left(\beta_{2}(t) y(t)+\beta_{3}(t) H(t)\right) S(t), \\
\frac{d I(t)}{d t}= & \beta_{2}(t) y(t) S(t)-(\mu(t)+d(t)) I(t), \\
\frac{d H(t)}{d t}= & \beta_{3}(t) S(t) H(t) \\
& -(\mu(t)+\alpha(t)+\gamma(t)) H(t), \\
\frac{d R(t)}{d t}= & \gamma(t) H(t)-\mu(t) R(t), \\
& S(0)>0, I(0) \geq 0, H(0) \geq 0, R(0) \geq 0 .
\end{aligned}
$$

Let $(x(t), y(t))$ be the solutions for model (51) and $(S(t), I(t), H(t), R(t))$ the solutions for model (52). We consider $y$ in system (51) and $H$ in system (52) for the persistence, permanence, and extinction of the infectives. We have the following definitions.

If

$$
\begin{gathered}
\liminf _{t \rightarrow \infty} y(t)>0 \\
\left(\liminf _{t \rightarrow \infty} H(t)>0\right),
\end{gathered}
$$

then we say that the infectives $y(H)$ are strongly persistent. 
If there are constants $w_{2}>w_{1}>0\left(v_{2}>v_{1}>0\right)$ such that

$$
\begin{gathered}
w_{1} \leq \liminf _{t \rightarrow \infty} y(t) \leq \limsup _{t \rightarrow \infty} y(t) \leq w_{2}, \\
\left(v_{1} \leq \liminf _{t \rightarrow \infty} H(t) \leq \limsup _{t \rightarrow \infty} H(t) \leq v_{2},\right)
\end{gathered}
$$

we say that the infectives $y(H)$ are permanent, if $y(t) \rightarrow 0$ as $t \rightarrow \infty$ for any solution $(x(t), y(t))$ of model (51), ((S $(t)$, $I(t), H(t), R(t))$ of model (52) with initial values $x\left(t_{0}\right)>0$, $y\left(t_{0}\right)>0(S(0)>0, I(0) \geq 0, H(0) \geq 0$, and $R(0) \geq 0)$; for some $t_{0} \geq 0$, we say that infectives $y(H)$ go extinct.

For model (51), we introduce the following assumption.

$\left(H_{1}\right)$ Functions $\lambda(t), b(t), \beta_{1}(t), m(t)\left(\Lambda(t), \mu(t), \beta_{2}(t)\right.$, $\left.\beta_{3}(t), d(t), \alpha(t), \gamma(t)\right)$ are nonnegative, continuous, and bounded on $R_{+}$.

$\left(H_{2}\right)$ There exist positive constants $w_{i}(i=1,2)$, such that

$$
\begin{aligned}
& \liminf _{t \rightarrow \infty} \int_{t}^{t+w_{1}} \lambda(s) d s>0, \\
& \liminf _{t \rightarrow \infty} \int_{t}^{t+w_{2}} b(s) d s>0, \\
& \liminf _{t \rightarrow \infty} \int_{t}^{t+w_{3}} m(s) d s>0 . \\
& \left(\liminf _{t \rightarrow \infty} \int_{t}^{t+w_{1}} \Lambda(s) d s>0, \liminf _{t \rightarrow \infty} \int_{t}^{t+w_{2}} \mu(s) d s\right. \\
& >0, \liminf _{t \rightarrow \infty}^{t+w_{3}} d(s) d s \\
& >0, \liminf _{t \rightarrow \infty} \int_{t}^{t+w_{4}} \alpha(s) d s \\
& \left.>0, \liminf _{t \rightarrow \infty} \int_{t}^{t+w_{5}} \gamma(s) d s>0 .\right)
\end{aligned}
$$

It is easy to prove that assumption $\left(\mathrm{H}_{2}\right)$ is equivalent to

$$
\begin{aligned}
& \liminf _{t, s \rightarrow \infty} \frac{1}{t} \int_{0}^{t} \lambda(r+s) d s>0, \\
& \liminf _{t, s \rightarrow \infty} \frac{1}{t} \int_{0}^{t} b(r+s) d s>0, \\
& \liminf _{t, s \rightarrow \infty} \frac{1}{t} \int_{0}^{t} m(r+s) d s>0, \\
& \left(\liminf _{t, s \rightarrow \infty} \frac{1}{t} \int_{0}^{t} \Lambda(r+s) d s\right. \\
& >0, \liminf _{t, s \rightarrow \infty} \frac{1}{t} \int_{0}^{t} \mu(r+s) d s>0,
\end{aligned}
$$

$$
\begin{aligned}
& \left(\liminf _{t, s \rightarrow \infty} \frac{1}{t} \int_{0}^{t} d(r+s) d s\right. \\
& >0, \liminf _{t, s \rightarrow \infty} \frac{1}{t} \int_{0}^{t} \alpha(r+s) d s \\
& \left.>0, \liminf _{t, s \rightarrow \infty} \frac{1}{t} \int_{0}^{t} \gamma(r+s) d s>0 .\right)
\end{aligned}
$$

In particular, when system (51) degenerates into $w$-periodic system, that is, $\lambda(t), b(t), \beta_{1}(t)$ and $m(t)(\Lambda(t), \mu(t), d(t), \alpha(t)$ and $\gamma(t))$ are all nonnegative continuous periodic functions with period $w>0$, then $\left(\mathrm{H}_{2}\right)$ is equivalent to the following cases: $\bar{\lambda}>0$ and $\bar{b}>0(\bar{\Lambda}>0, \bar{\mu}>0, \bar{d}>0, \bar{\alpha}>0$, and $\bar{\gamma}>0)$. Let us denote the average value of $g(t)$ by $m(g)$ for any continuous periodic function $g(t)$; that is,

$$
m(g)=\lim _{t \rightarrow \infty} \frac{1}{t} \int_{0}^{t} g(s) d s .
$$

Then we consider the following nonautonomous linear equations:

$$
\begin{gathered}
\frac{d z}{d t}=\lambda(t)-b(t) z, \\
\left(\frac{d z}{d t}=\Lambda(t)-\mu(t) z\right) .
\end{gathered}
$$

We have the following results.

Theorem 8. Suppose that assumptions $\left(H_{1}\right)$ and $\left(H_{2}\right)$ hold; then consider the following.

(a) Each fixed solution $z^{*}(t)$ of (58) (see (59)) with initial value $z^{*}(0)>0$ is bounded and globally uniformly attractive on $\left[t_{0}, \infty\right)$.

(b) Let $z(t)$ be a solution of (58) (see (59)) and $\overline{z(t)} a$ solution obtained in (58) (see (59)); when $\lambda(t)(\Lambda(t))$ is replaced by another continuous function $\overline{\lambda(t)}(\overline{\Lambda(t)})$ and $z\left(t_{0}\right)=\overline{z\left(t_{0}\right)}$, then there is a constant $L>0,\left(L^{\prime}>0\right)$ depending only on $b(t)(\mu(t))$ such that $\sup _{t \geq t_{0}}|z(t)-\overline{z(t)}| \leq L \sup _{t \geq t_{0}} \mid \lambda(t)-$ $\overline{\lambda(t)} \mid\left(\sup _{t \geq t_{0}}|z(t)-\overline{z(t)}| \leq L^{\prime} \sup _{t \geq t_{0}}|\Lambda(t)-\overline{\Lambda(t)}|\right)$.

(c) If there is a constant $w_{0}>0$ such that

$$
\begin{gathered}
\liminf _{t \rightarrow \infty} \int_{t}^{t+w_{0}} \lambda(s) d s>0 \\
\left(\liminf _{t \rightarrow \infty} \int_{t}^{t+w_{0}} \Lambda(s) d s>0\right),
\end{gathered}
$$

then there are constants $0<m<M$ such that

$$
m \leq \liminf _{t \rightarrow \infty} z(t) \leq \limsup _{t \rightarrow \infty} z(t) \leq M
$$

for any solution $z(t)$ of (58) (see (59)) with initial value $z\left(t_{0}\right)>$ 0 .

(d) When (58) (see (59)) is w-periodic, then (58) (see (59)) has a unique nonnegative almost periodic solution $z^{*}(t)$ which is globally uniformly attractive. 
(e) When (58) (see (59)) is almost periodic, then (58) (see (59)) has a unique nonnegative almost periodic solution $z^{*}(t)$ which is globally uniformly attractive.

(f) For bird system, if

$$
\liminf _{t \rightarrow \infty} b(t)>0
$$

then for any solution $z(t)$ of (58) with the initial value $z\left(t_{0}\right)>0$

$$
\left(\frac{\lambda}{b}\right)_{m} \leq \liminf _{t \rightarrow \infty} z(t) \leq \limsup _{t \rightarrow \infty} z(t) \leq\left(\frac{\lambda}{b}\right)_{M},
$$

where

$$
\begin{aligned}
& \left(\frac{\lambda}{b}\right)_{m}=\liminf _{t \rightarrow \infty} \frac{\lambda(t)}{b(t)}, \\
& \left(\frac{\lambda}{b}\right)_{M}=\limsup _{t \rightarrow \infty} \frac{\lambda(t)}{b(t)} .
\end{aligned}
$$

For human system, if $\mu(t)>0$ for $t>0$ and $0<$ $\liminf _{t \rightarrow \infty}(\Lambda(t) / \mu(t)) \leq \limsup _{t \rightarrow \infty}(\Lambda(t) / \mu(t))<\infty$, then for any solution $z(t)$ of (59) with initial value $z(0)>0$, we have

$$
\left(\frac{\Lambda}{\mu}\right)_{m} \leq \liminf _{t \rightarrow \infty} z(t) \leq \limsup _{t \rightarrow \infty} z(t) \leq\left(\frac{\Lambda}{b}\right)_{M},
$$

where

$$
\begin{aligned}
& \left(\frac{\Lambda}{\mu}\right)_{m}=\liminf _{t \rightarrow \infty} \frac{\Lambda(t)}{\mu(t)}, \\
& \left(\frac{\Lambda}{\mu}\right)_{M}=\limsup _{t \rightarrow \infty} \frac{\Lambda(t)}{\mu(t)} .
\end{aligned}
$$

5.1. Bird World. Using the variation of constants method, comparison theorem, and the method of Lyapunov function, we can prove this lemma very easily; here, we omit it.

Let $b(t, u)=\beta_{1}(t) u-(b(t)+m(t))$, and let $z^{*}(t)$ be some fixed solution of (58) with initial value $z^{*}(0)>0$. Firstly, on the persistence and permanence of infective $y$ in model (51), we have the following theorem.

Theorem 9. Suppose that $\left(H_{1}\right)$ and $\left(H_{2}\right)$ hold. Then the following statements are equivalent for model (51):

(a) Infective $y$ is permanent.

(b) Infective $y$ is strongly persistent.

(c) There is a constant $\alpha>0$ such that

$$
R_{0}^{\star}=\liminf _{t \rightarrow \infty} \int_{t}^{t+\alpha} b\left(s, z^{*}(s)\right) d s>0 .
$$

Proof. Let $n_{1}(t)=x(t)+y(t)$ with the initial value $n_{1}(0)=x(0)+y(0)$. Firstly, we prove that the number $R_{0}^{\star}$ is independent of the choice of $z^{*}(t)$. In fact, by (a) of Theorem 8 , for arbitrary small $\epsilon>0$ and any solution $z(t)$ of (58) with initial value $z(0)>0$, there exists $T>0$ such that

$$
z^{*}(t)-\epsilon \leq z(t) \leq z^{*}(t)+\epsilon
$$

for all $t \geq T$. Hence, $b\left(t, z^{*}(t)-\epsilon\right) \leq b(t, z(t)) \leq b\left(t, z^{*}(t)+\epsilon\right)$ for all $t \geq T$. Since

$$
\begin{aligned}
& \liminf _{t \rightarrow \infty} \int_{t}^{t+\alpha} b\left(s, z^{*}(s)+\epsilon\right) d s \leq R_{0}^{\star}+\sup _{t \geq 0} \beta_{1}(t) \alpha \epsilon, \\
& \liminf _{t \rightarrow \infty} \int_{t}^{t+\alpha} b\left(s, z^{*}(s)-\epsilon\right) d s \geq R_{0}^{\star}-\sup _{t \geq 0} \beta_{1}(t) \alpha \epsilon,
\end{aligned}
$$

we have

$$
\begin{aligned}
R_{0}^{\star}-\sup _{t \geq 0} \beta_{1}(t) \alpha \epsilon & \leq \liminf _{t \rightarrow \infty} \int_{t}^{t+\alpha} b(s, z(s)) d s \\
& \leq R_{0}^{\star}+\sup _{t \geq 0} \beta_{1}(t) \alpha \epsilon .
\end{aligned}
$$

From the arbitrariness of $\epsilon$, we finally obtain

$$
R_{0}^{\star}=\liminf _{t \rightarrow \infty} \int_{t}^{t+\alpha} b(s, z(s)) d s .
$$

This shows that $R_{0}^{\star}$ is independent of the choice of $z^{*}(t)$. Let $X(t)=\left(n_{1}(t), y(t)\right)$ be any solution of system (51) with $n_{1}\left(t_{0}\right) \geq 0$ and $y\left(t_{0}\right) \geq 0$ for some $t_{0} \geq 0$. We next prove that $x(t)$ and $y(t)$ are nonnegative and $x(t)$ is ultimately bounded on $\left[t_{0}, \infty\right)$. In fact, from the second equation of system (51), we have

$$
\begin{aligned}
y(t) & =y\left(t_{0}\right) \exp \int_{t_{0}}^{t}\left[b\left(s, n_{1}(s)\right)-\beta_{1}(s) y(s)\right] d s \\
& \geq 0
\end{aligned}
$$

for all $t \geq t_{0}$. Hence, $y(t)$ is nonnegative on $\left[t_{0}, \infty\right)$.

Similarly

$$
x^{\prime}=\lambda(t)-b(t) x(t)-\beta_{1}(t) x(t) y(t) .
$$

We have

$$
x(t) \geq x\left(t_{0}\right) \exp \int_{t_{0}}^{t}\left[-\left(b(s)+\beta_{1}(s) y(s)\right)\right] d s \geq 0
$$

for all $t \geq t_{0}$ since $n_{1}^{\prime}(t)=\lambda(t)-b(t) n_{1}(t)-m(t) y(t) \leq$ $\lambda(t)-b(t) n_{1}(t)$ for all $t \geq t_{0}$. Using the comparison theorem and conclusion (a) of Theorem 8 , we can obtain that there is a constant $M_{1}>0$, such that for any solution $(x(t), y(t))$ of system (51) there is $t_{1}>t_{0}$ such that $n_{1}(t)<M_{1}$ for all $t>t_{1}$. Since $n_{1}(t)=x(t)+y(t)$, we further obtain $x(t)<M_{1}$ and $y(t)<M_{1}$ for $t>t_{1}$. Therefore, all solutions $(x(t), y(t))$ of system (51) with initial values $x\left(t_{0}\right) \geq 0$ and $y\left(t_{0}\right) \geq 0$ for some $t_{0} \geq 0$ are ultimately bounded. Now, we prove (c) $\Rightarrow$ (a). If (c) is true, then, by $\left(H_{1}\right)$ and $\left(H_{2}\right)$, we can choose small enough positive constants $\epsilon_{1}, \xi, \eta$ and a large enough constant $T_{0} \geq 0$, such that

$$
\begin{gathered}
\int_{t}^{t+\alpha}\left[b\left(s, z^{*}(s)-\xi\right)-\beta_{1}(s) \epsilon_{1}\right] d s>\eta \\
\int_{t}^{t+w_{2}}\left[\beta_{1}(s) M \epsilon_{1}-(b(s)+m(s)) \epsilon_{1}\right] d s<-\eta
\end{gathered}
$$

for all $t \geq T_{0}$. 
For any positive constant $\epsilon_{2}>0$, we consider the following equation:

$$
\frac{d u}{d t}=\lambda(t)-b(t) u-m(t) \epsilon_{2}
$$

for any $\tau_{0} \in R_{+}$and $u_{0} \in R_{+}$. Let $u(t)$ be the solution of (58) with the initial value $u\left(\tau_{0}\right)=u_{0}$ and $\overline{z(t)}$ the solution of (58) with initial value $\overline{z\left(\tau_{0}\right)}=u_{0}$. By conclusion (b) of Theorem 9 there is a constant $L>0$, depending only on $b(t)$, such that

$$
|u(t)-\bar{z}(t)|<L \sup _{t \geq t_{0}}|m(t)| \epsilon_{2}
$$

for all $t \geq \tau_{0}$. We choose sufficiently small $\epsilon_{2} \in\left(0, \epsilon_{1}\right)$ and $\epsilon_{2}$ is independent of any $\tau_{0}$ and $u_{0}$, such that

$$
|u(t)-\overline{z(t)}|<\frac{1}{2} \xi
$$

for all $t \geq \tau_{0}$. By conclusion (a) of Theorem 9 we know that $z^{*}(t)$ is globally uniformly attractive on $R_{+}$. Thus, for every constant $\xi$, there is $T_{1}>0$, such that

$$
\left|\overline{z(t)}-z^{*}(t)\right|<\frac{1}{2} \xi
$$

for all $t \geq \tau_{0}+T_{1}$ and for some $t_{0} \geq 0$. Let $(x(t), y(t))$ be any solution of system (51) with initial values $x\left(t_{0}\right)>0$ and $y\left(t_{0}\right)>0$ for some $t_{0} \geq 0$. Then, we prove

$$
\limsup _{t \rightarrow \infty} y(t) \geq \epsilon_{2} \text {. }
$$

Suppose that (81) is not true; there exists $T_{2} \geq t_{0}$ such that $y(t)<\epsilon_{2}$ for all $t>T_{2}$. From the first equation of system (51), we have

$$
\begin{aligned}
n_{1}^{\prime}(t) & =\lambda(t)-b(t) n_{1}(t)-m(t) y(t) \\
& \geq \lambda(t)-b(t) n_{1}(t)-m(t) \epsilon_{2}
\end{aligned}
$$

for all $t \geq T_{2}$. Let $u(t)$ be the solution of (58) with initial value $u\left(T_{2}\right)=n_{1}\left(T_{2}\right)$. By comparison theorem we have $n_{1}(t) \geq u(t)$ for all $t \geq T_{2}$. From (79) for $\tau_{0}=T_{2}$, we can obtain $\mid u(t)-$ $\overline{z(t)} \mid<(1 / 2) \xi$ for all $t \geq T_{2}$. By (76), we have

$$
n_{1}(t) \geq u(t)>\overline{z(t)}-\frac{\xi}{2}>z^{*}-\xi
$$

for all $t \geq T_{3}$, where $T_{3}=\max \left\{T_{0}, T_{1}, T_{2}\right\}$. Integrating the second equation of system (51) from $t_{1}$ to $t$ and using (75) and (83), we have

$$
\begin{aligned}
y(t) & =y\left(t_{1}\right) \exp \int_{t_{1}}^{t}\left[b(s, n(s))-\beta_{1}(s) y(s)\right] d s \\
& \geq y\left(t_{1}\right) \exp \int_{t_{1}}^{t}\left[b\left(s, z^{*}(s)-\xi\right)-\beta_{1}(s) \epsilon_{1}\right] d s
\end{aligned}
$$

for all $t \geq T_{3}$. By (75) we have $y(t) \rightarrow \infty$ as $t \rightarrow \infty$. This is contradictory with $y(t)<\epsilon_{2}$ for all $t \geq T_{3}$. So we conclude that $\limsup _{t \rightarrow \infty} y(t) \geq \epsilon_{2}$. Then we prove that there exists $v>0$ such that

$$
\liminf _{t \rightarrow \infty} y(t)>v \text {. }
$$

In fact, from (75) and (76) we can obtain that there is a positive constant $P$ such that

$$
\begin{gathered}
\int_{t}^{t+\beta}\left[b\left(s, z^{*}(s)-\xi\right)-\beta_{1}(s) \epsilon_{1}\right] d s>\eta, \\
\int_{t}^{t+\beta}\left[\beta_{1}(s) M \epsilon_{1}-(b(s)+m(s)) \epsilon_{1}\right] d s<-M
\end{gathered}
$$

for all $t \geq T_{0}$ and $\beta>P$, where the constant $M=M_{1}$; we assume that there is a sequence of initial value $x_{n}^{\prime}=$ $\left(x_{n}, y_{n}\right)(n=1,2, \ldots)$ with $x_{n}>0$ and $y_{n}>0$ such that

$$
\liminf _{t \rightarrow \infty} y\left(t, x_{n}^{\prime}\right)<\frac{\epsilon_{2}}{n^{2}}, \quad n=1,2, \ldots
$$

From $\lim \sup _{t \rightarrow \infty} y(t) \geq \epsilon_{2}$, for every $n$ there are two time sequences $T_{q}^{(n)}$ and $S_{q}^{(n)}$ satisfying

$$
T_{0}<S_{1}^{(n)}<T_{1}^{(n)}<S_{2}^{(n)}<T_{2}^{(n)} \cdots<S_{q}^{(n)}<T_{q}^{(n)}<\cdots
$$

and $\lim _{n \rightarrow \infty} S_{q}^{(n)}=\infty$, such that

$$
\begin{aligned}
y\left(S_{q}^{(n)}, x_{n}^{\prime}\right) & =\frac{\epsilon_{2}}{n}, \\
y\left(T_{q}^{(n)}, x_{n}^{\prime}\right) & =\frac{\epsilon_{2}}{n^{2}} \\
\frac{\epsilon_{2}}{n^{2}} & <y\left(t, x_{n}^{\prime}\right)<\frac{\epsilon_{2}}{n}
\end{aligned}
$$

for all $t \in\left(S_{q}^{(n)}, T_{q}^{(n)}\right)$. By the ultimate boundedness of solution of system (42), for each $n$ we can choose positive integer $K^{(n)}$ such that $n\left(t, x_{n}^{\prime}\right)<M, y\left(t, x_{n}^{\prime}\right)<M, n=1,2, \ldots$, for all $q>K^{(n)}$ and $t \in\left(S_{q}^{(n)}, T_{q}^{(n)}\right)$. Let $q \geq K^{(n)}$, and then, for any $t \in\left(S_{q}^{(n)}, T_{q}^{(n)}\right)$, we have

$$
\begin{aligned}
& \frac{d y\left(t, x_{n}\right)}{d t} \\
& \quad=y\left(t, x_{n}\right)\left[b\left(t, n_{1}\left(t, x_{n}\right)\right)-\beta_{1}(t) y\left(t, x_{n}\right)\right] \\
& \geq y\left(t, x_{n}\right)\left[-|b(t, M)|-\beta_{1}(t) M\right] \geq-\gamma y\left(t, x_{n}\right),
\end{aligned}
$$

where $\gamma=\sup _{t \geq 0}|b(t, M)|+\beta_{1}(t) M$.

Integrating the inequality from $S_{q}^{(n)}$ to $T_{q}^{(n)}$, we have

$$
y\left(T_{q}^{(n)}, x_{n}\right) \geq y\left(S_{q}^{(n)}, x_{n}\right) \exp \left[-\gamma\left(T_{q}^{(n)}-S_{q}^{(n)}\right)\right] .
$$

By (79), we have

$$
\frac{\epsilon_{2}}{n^{2}} \geq \frac{\epsilon_{2}}{n} \exp \left[-\gamma\left(T_{q}^{(n)}-S_{q}^{(n)}\right)\right] .
$$

Hence,

$$
T_{q}^{(n)}-S_{q}^{(n)} \geq \frac{\ln n}{\gamma} \longrightarrow \infty, \quad q>K^{(n)}
$$


For each $n$, from the first equation of system (51), it follows that

$$
\begin{aligned}
n_{1}^{\prime}(t) & =\lambda(t)-b(t) n_{1}\left(t, x_{n}^{\prime}\right)-\beta_{1}(t) y\left(t, x_{n}^{\prime}\right) \\
& \geq \lambda(t)-b(t) n_{1}\left(t, x_{n}^{\prime}\right)-\beta_{1}(t) \epsilon_{2}
\end{aligned}
$$

for all $t \in\left[S_{q}^{(n)}, T_{q}^{(n)}\right]$ and $q>K^{(n)}$. Let $u(t)$ be the solution of (77) with initial value $u\left(S_{q}^{(n)}\right)=n\left(S_{q}^{(n)}, x_{n}^{\prime}\right)$, and by the comparison theorem we have $n\left(t, x_{n}^{\prime}\right) \geq u(t)$ for all $t \in$ $\left[S_{q}^{(n)}, T_{q}^{(n)}\right]$ and $q>K^{(n)}$.

From (79), for $\tau_{0}=S_{q}^{(n)}$ and $u_{0}=n_{1}\left(S_{q}^{(n)}, x_{n}^{\prime}\right)$, we have

$$
|u(t)-\overline{z(t)}|<\frac{1}{2} \xi
$$

for all $t \in\left[S_{q}^{(n)}, t_{q}^{(n)}\right]$, where $\overline{z(t)}$ is the solution of (58) with the initial value $\overline{z\left(S_{q}^{(n)}\right)}=n_{1}\left(S_{q}^{(n)}, x_{n}\right)$. Since $z^{*}(t)$ is globally uniformly attractive on $R_{+}$for (58) there is a constant $T^{*}>0$ and $T^{*}$ is independent of any $n$ and $q$, such that

$$
\left|\overline{z(t)}-z^{*}(t)\right|<\frac{1}{2} \xi
$$

for all $t \geq S_{q}^{(n)}+T^{*}$. From (93), we can choose a large enough integer $B$ such that

$$
T_{q}^{(n)}-S_{q}^{(n)}>2 P+T^{*}
$$

for all $n \geq B$ and $q>K^{(n)}$. Hence, by (95) and (96), when $n \geq B$ and $q>K^{(n)}$, for any $t \in\left[S_{q}^{(n)}+T^{*}, T_{q}^{(n)}\right]$, we have

$$
n_{1}\left(t, x_{n}^{\prime}\right) \geq u(t)>\overline{z(t)}-\frac{\xi}{2}>z^{*}(t)-\xi
$$

Integrating the second equation of system (51) from $\bar{t}+P+T^{*}$ to $T_{q}^{(n)}$ and having $\bar{t} \in\left(S_{q}^{(n)}, S_{q}^{(n)}+P\right)$, we finally have

$$
\begin{aligned}
\frac{\epsilon_{2}}{n^{2}} & =y\left(T_{q}^{(n)}, x_{n}^{\prime}\right)=y\left(\bar{t}, x_{n}^{\prime}\right) \\
& \cdot \exp \int_{\bar{t}+P+T^{*}}^{T_{q}^{(n)}}\left[b\left(t, n\left(t, x_{n}^{\prime}\right)\right)-\beta_{1}(t) y\left(t, x_{n}^{\prime}\right)\right] d t \\
& \geq \frac{\epsilon_{2}}{n^{2}} \exp \int_{\bar{t}+P+T^{*}}^{T_{q}^{(n)}}\left[b\left(t, z^{*}(t)-\xi\right)-\beta_{1}(t) \epsilon_{1}\right] d t \\
& >\frac{\epsilon_{2}}{n^{2}} .
\end{aligned}
$$

This leads to a contradiction. Thus, we finally prove that inequality (85) is true. At last, we prove that (b) $\Rightarrow$ (c). In fact, if (c) is not true, then we have

$$
\liminf _{t \rightarrow \infty} \int_{t}^{t+\alpha} b\left(s, z^{*}(s)\right) d s \leq 0
$$

for any constant $\alpha>0$. Let $(x(t), y(t))$ be the solution of system (51) with the initial values $z(0)=z^{*}(0)$ and $y(0)>0$.
Since $\liminf \operatorname{in}_{t \rightarrow \infty} y(t)>0$, there is a constant $\delta>0$ such that $y(t) \geq \delta$ for all $t \geq 0$. Letting $M_{0}=\sup _{t \geq 0} y(t)$, then $0<M_{0}<\infty$. Since $\liminf _{t \rightarrow \infty} \int_{t}^{t+w_{1}} \beta_{1}(s) d s>0$, there are constants $\gamma_{0}>0$ and $T \geq 0$, such that for $t \geq T$ we have $\int_{t}^{t+w_{1}} \beta_{1}(s) d s \geq \gamma_{0}$. We choose a constant $P>0$, such that $M_{0} \exp (-(1 / 2) P \delta)<\delta$. For this constant $P$, we further choose an integer $K_{P}>0$ such that

$$
K_{P} \gamma_{0}-\sup _{t \geq 0} \beta_{1}(t) w_{1} \geq P
$$

Thus, for any $\theta \geq K_{P} w_{1}$ and $t \geq T$, since there is an integer $K \geq K_{P}$, such that $\theta \in\left[K w_{1},(K+1) w_{1}\right]$, we obtain

$$
\begin{aligned}
\int_{t}^{t+\theta} & \beta_{1}(s) d s \\
= & {\left[\int_{t}^{t+w_{1}}+\cdots+\int_{t+(K-1) w_{1}}^{t+K w_{1}}+\int_{t+K w_{1}}^{t+\theta}\right] \beta_{1}(s) d s } \\
\geq & K \gamma_{0}-\sup _{t \geq 0} \beta_{1}(t) w_{1} \geq P .
\end{aligned}
$$

From this inequality and by (100), for any constant $\alpha \geq K_{p} w_{1}$, we have

$$
\liminf _{t \rightarrow \infty} \int_{t}^{t+\alpha}\left[b\left(s, z^{*}(s)\right)-\beta_{1}(s) y(s)\right] d s \leq-P \delta .
$$

Hence, there is $t^{*} \geq r$, such that

$$
\int_{t^{*}}^{t^{*}+\alpha}\left[b\left(s, z^{*}(s)\right)-\beta_{1}(s) y(s)\right] d s \leq-\frac{1}{2} P \delta .
$$

Since $n_{1}^{\prime}(t)=\lambda(t)-b(t) n(t)-m(t) y(t) \leq \lambda(t)-b(t) n_{1}(t)$ for all $t \geq 0$, by $n_{1}(0)=z^{*}(0)$ and comparison theorem,

$$
\begin{aligned}
& y\left(t^{*}+\alpha\right) \\
& \quad=y\left(t^{*}\right) \exp \int_{t^{*}}^{t^{*}+\alpha}\left[b\left(s, z^{*}(s)\right)-\beta_{1}(s) \delta\right] d s .
\end{aligned}
$$

Now using (104), we obtain $\delta \leq M_{0} \exp (-(1 / 2) P \delta)<\delta$, which leads to a contradiction. This shows that if (b) holds, then (c) also holds, since (a) $\Rightarrow(\mathrm{b})$ is obvious. This completes the proof of Theorem 9 .

Theorem 10. Suppose that assumptions $\left(H_{1}\right)$ and $\left(H_{2}\right)$ hold and infective $y$ is permanent in model (51). Then susceptible $x$ in model (51) is also permanent.

This theorem can be proved easily by using the similar method given in the proof of Theorem 9.

Theorem 11. Suppose that assumptions $\left(H_{1}\right)$ and $\left(H_{2}\right)$ hold. If there is a constant $\alpha>0$, such that

$$
\begin{aligned}
R_{1}^{*} & =\limsup _{t \rightarrow \infty} \int_{t}^{t+\alpha} b\left(s, z^{*}(s)\right) d s \leq 0 \\
\text { or } R_{2}^{*} & =\limsup _{t \rightarrow \infty} \frac{1}{t} \int_{0}^{t} b\left(s, z^{*}(s)\right) d s<0,
\end{aligned}
$$

then infective $y$ in model (51) is extinct. 
Proof. If (106) holds, then by assumptions $\left(H_{1}\right)$ and $\left(H_{2}\right)$, for any constant $\epsilon \in(0,1)$, we can choose constants $\epsilon^{\prime} \in(0, \epsilon)$, $\delta>0$, and positive constant $T_{0}$ which is large enough such that

$$
\int_{t}^{t+\lambda}\left[b\left(s, z^{*}(s)+\epsilon^{\prime}\right)-\beta_{1}(s) \epsilon\right] d s<-\delta
$$

for all $t \geq T_{0}$. If (107) holds, then by assumptions $\left(H_{1}\right)$ and $\left(H_{2}\right)$ we can directly choose $\epsilon^{\prime}, \delta$, and $T_{0}$, where $\epsilon^{\prime}, \delta$ are small enough and $T_{0}$ is large enough, such that

$$
\frac{1}{t} \int_{0}^{t} b\left(s, z^{*}(s)+\epsilon^{\prime}\right) d s<-\delta
$$

for all $t \geq T_{0}$.

For any solution $(x(t), y(t))$ of system (51) with initial values $x\left(t_{0}\right)>0$ and $y\left(t_{0}\right)>0$ for some $t_{0} \geq 0$, since $n_{1}^{\prime}(t)=\lambda(t)-b(t) n_{1}(t)-m(t) y(t) \leq \lambda(t)-b(t) n_{1}(t)$ for all $t \geq t_{0}$, we have $n_{1}(t) \leq z(t)$ for all $t \geq t_{0}$, where $z(t)$ is the solution of (58) with the initial value $z\left(t_{0}\right)=n_{1}\left(t_{0}\right)$. Since $z^{*}(t)$ is uniformly globally attractive on $R_{+}$for $(58)$, we obtain that, for $\varepsilon^{\prime}>0$ given above, there exists $T_{1}>T_{0}$, such that

$$
\left|z(t)-z^{*}(t)\right|<\epsilon^{\prime}
$$

for all $t \geq T_{1}$. Hence, we have $n_{1} \leq z(t)<z^{*}(t)+\epsilon^{\prime}$ for all $t \geq T_{1}$.

Since

$$
\begin{aligned}
y^{\prime}(t) & =y(t)\left[b\left(t, n_{1}(t)\right)-\beta_{1}(t) y(t)\right] \\
& \leq y(t)\left[b\left(t, z^{*}(t)+\epsilon^{\prime}\right)-\beta_{1}(t) y(t)\right]
\end{aligned}
$$

for all $t \geq T_{1}$, by integrating we further have

$$
\begin{aligned}
& y(t) \\
& \quad \leq y\left(T_{1}\right) \exp \int_{t_{1}}^{t}\left[b\left(s, z^{*}(s)+\epsilon^{\prime}\right)-\beta_{1}(s) y(s)\right] d s
\end{aligned}
$$

for all $t \geq T_{1}$. Suppose $R_{1}^{*} \leq 0$ holds. If $y(t) \geq \epsilon$ for all $t \geq T_{1}$, then from (112) we obtain

$$
y(t) \leq y\left(T_{1}\right) \exp \int_{T_{1}}^{t}\left[b\left(s, z^{*}(s)+\epsilon^{\prime}\right)-\beta_{1}(s) \epsilon\right] d s
$$

By (109), it follows that $y(t) \rightarrow-\infty$ as $t \rightarrow \infty$. This leads to a contradiction. Hence, there exists $t_{1}>T_{1}$, such that $y\left(t_{1}\right)<\epsilon$. Letting

$$
n(\epsilon)=\sup _{t \geq T_{1}}\left[\left|b\left(s, z^{*}(t)+\epsilon^{\prime}\right)\right|+\beta_{1}(t) \epsilon\right]
$$

then $n(\epsilon)$ is bounded for all $0 \leq \epsilon \leq 1$. We will prove

$$
y(t)<\epsilon \exp (n(\epsilon) \lambda)
$$

for all $t \geq t_{1}$.
If (115) is not true, then there is $t_{2}>t_{1}$, such that $y(t)>$ $\epsilon \exp (n(\epsilon) \lambda)$. Hence, there exists $t_{3} \in\left(t_{1}, t_{2}\right)$, such that $y\left(t_{3}\right)=$ $\epsilon$ and $y(t)>\epsilon$ for all $t \in\left\{t_{3}, t_{2}\right\}$. Let $P$ be a nonnegative integer such that

$$
t_{2} \in\left(t_{3}+p \lambda, t_{3}+(p+1) \lambda\right) .
$$

Then from (110) and (113) we have

$$
\begin{aligned}
& \epsilon \exp \left(n_{1}(\epsilon) \lambda\right)<y\left(t_{2}\right) \\
& \quad=y\left(t_{3}\right) \exp \int_{t_{3}}^{t_{2}}\left[b\left(s, n_{1}(s)\right)-\beta_{1}(s) y(s)\right] d s \\
& \quad \leq \epsilon \exp \int_{t_{3}}^{t_{2}}\left[b\left(s, z^{*}(s)+\epsilon^{\prime}\right)-\beta_{1}(s) \epsilon\right] d s \\
& \quad<\epsilon \exp \left(n_{1}(\epsilon) \lambda\right) .
\end{aligned}
$$

This leads to a contradiction. Hence (115) holds. Furthermore, as $\epsilon$ is arbitrary, we conclude that $y(t) \rightarrow 0$ as $t \rightarrow \infty$. Supposing that (107) holds, then from (113) we get

$$
y(t) \leq y\left(T_{1}\right) \exp \int_{t}^{T_{1}} b\left(s, z^{*}(s)+\epsilon^{\prime}\right) d s
$$

for all $t \geq T_{1}$. From (109), we have $y(t) \rightarrow 0$ as $t \rightarrow \infty$.

5.2. Human World ( $\epsilon=0, H \neq 0$ ). In this section, we will give conditions under which the solutions exist on $[0,+\infty)$ and are positive. The main result is as follows.

Theorem 12. Suppose that assumptions $\left(H_{1}\right)$ and $\left(H_{2}\right)$ hold. The solution $(S(t), I(t), H(t), R(t))$ with initial conditions $S(0)>0, I(0)>0, H(0)>0$, and $R(0)>0$ is nonnegative and uniformly bounded on $[0,+\infty)$. define

We can easily prove this theorem using Theorem 1. We

$$
\begin{aligned}
& a=\sup _{t \geq 0} \beta_{2}(t), \\
& b=\sup _{t \geq 0} \alpha(t), \\
& c=\sup _{t \geq 0} \mu(t), \\
& e=\sup _{t \geq 0} \gamma(t) .
\end{aligned}
$$

In this section, we will discuss the permanence of the disease for system (52) and will demonstrate how the disease for system (58) will be permanent under certain conditions. Let the function

$$
b(t, u)=\beta_{3}(t) u-(\alpha(t)+\alpha(t)+\gamma(t)) .
$$

And let $z^{*}(t)$ be some fixed solution of (59) with initial value $z^{*}(0)>0$. We have the following theorem.

Theorem 13. Suppose that assumptions $\left(H_{1}\right)$ and $\left(H_{2}\right)$ hold and there is a constant $\lambda^{\prime}>0$, such that

$$
R_{0}^{*}=\liminf _{t \rightarrow \infty} \int_{t}^{t+\lambda^{\prime}} b\left(s, z^{*}(s)\right) d s>0,
$$

and then the infective $H$ is permanent. 
Proof. Since $\varphi(S(t), H(t), R(t))$ is the solution of system (45), we know $n_{2}(t)$ is a solution of (59). System (52) is equivalent to the following system:

$$
\begin{aligned}
\frac{d I(t)}{d t}= & \beta_{2}(t)\left(n_{2}(t)-I(t)-H(t)-R(t)\right) y(t) \\
& -(\mu(t)+d(t)+\epsilon(t)) I(t), \\
\frac{d H(t)}{d t}= & \beta_{3}(t)\left(n_{2}(t)-I(t)-H(t)-R(t)\right) H(t) \\
& -(\mu(t)+\alpha(t)+\gamma(t)) H(t), \\
\frac{d R(t)}{d t}= & \gamma(t) H(t)-\mu(t) R(t) .
\end{aligned}
$$

Firstly, we prove that the number $R_{0}^{*}$ is independent of the choice of $z^{*}(t)$. In fact, Theorem 8 implies that, for any sufficiently small $\epsilon>0$ and any solution $z(t)$ of (59) with initial value $z(0)>0$, there exists $T>0$ such that $t \geq T$. Consider

$$
z^{*}(t)-\epsilon \leq z(t) \leq z^{*}(t)+\epsilon, \quad z^{*}(t) \geq m .
$$

Hence

$$
b\left(t, z^{*}(t)-\epsilon\right) \leq b(t, z(t)) \leq b\left(t, z^{*}(t)+\epsilon\right) .
$$

For $t \geq T$, we obtain

$$
\begin{gathered}
\liminf _{t \rightarrow \infty} \int_{t}^{t+\lambda^{\prime}} b\left(s, z^{*}(t)+\epsilon\right) d s \\
\leq R_{0}^{*}+\sup _{t \geq 0} \beta_{3}(t) \lambda^{\prime} \epsilon, \\
\liminf _{t \rightarrow \infty} \int_{t}^{t+\lambda^{\prime}} b\left(s, z^{*}(t)-\epsilon\right) d s \\
\geq R_{0}^{*}-\sup _{t \geq 0} \beta_{3}(t) \lambda^{\prime} \epsilon .
\end{gathered}
$$

We obtain

$$
\begin{aligned}
& \liminf _{t \rightarrow \infty} b\left(t, z^{*}(t)-\epsilon\right) \leq \liminf _{t \rightarrow \infty} \int_{t}^{t+\lambda^{\prime}} b(s, z(s)) d s \\
& \quad \leq \liminf _{t \rightarrow \infty} b\left(t, z^{*}(s)+\epsilon\right) d s .
\end{aligned}
$$

We finally obtain

$$
\liminf _{t \rightarrow \infty} \int_{t}^{t+\lambda^{\prime}} b(s, z(s)) d s=R_{0}^{*}
$$

So $R_{0}^{*}$ is independent of $z^{*}(t)$. Therefore,

$$
\liminf _{t \rightarrow \infty} \int_{t}^{t+\lambda^{\prime}} b\left(s, n_{2}(s)\right) d s>0 .
$$

By assumptions $\left(H_{1}\right),\left(H_{2}\right)$ and (128), we choose $\epsilon_{0}, \epsilon_{1}, \epsilon_{2}, \epsilon_{3}$, which are small enough positive constants; then there exist $T_{2}>0$ and $\eta_{1}>0$ satisfying

$$
\begin{array}{r}
\int_{t}^{t+w_{2}}\left[\beta_{2}(\theta) M \epsilon_{0}-(\mu(t)+d(t)) \epsilon_{1}\right] d \theta<-\eta_{1}, \\
\int_{t}^{t+w_{2}}\left[\gamma(\theta) \epsilon_{2}-\mu(t) \epsilon_{3}\right] d \theta<-\eta_{1}, \\
\int_{t}^{t+\lambda^{\prime}} b\left(s, n_{2}(s)-\epsilon_{1}-k \epsilon_{2}-\epsilon_{3}\right) d s>\eta_{1}, \\
n_{2}(t)-\epsilon_{1}-k \epsilon_{2}-\epsilon_{3} \geq m, \\
n_{2}(t) \leq M
\end{array}
$$

for all $t \geq T_{2}$, where $k=1+(a M+b) w_{2}$. Firstly, we will prove

$$
\limsup _{t \rightarrow \infty} H(t)>\epsilon_{2} \text {. }
$$

For any solution of (58), suppose that (133) is not true; then there exists a solution $(I(t), H(t), R(t))$ of system (58) and $T_{3}>T_{1}$ such that $H(t) \leq \epsilon_{2}$ for all $t \geq T_{3}$. If $I(t) \geq \epsilon_{1}$ for all $t \geq T_{3}$, then from the first equation of system (52) we have

$$
\begin{aligned}
I(t) & -I\left(T_{3}\right) \\
& =\int_{T_{3}}^{t} \beta_{2}(\theta)\left(n_{2}(\theta)-I(\theta)-H(\theta)-R(\theta)\right) y \\
& -(\mu(\theta)+d(\theta)) I(\theta) d \theta \leq \int_{T_{3}}^{t} \beta_{2}(\theta) M \epsilon_{0} \\
& -(\mu(\theta)+d(\theta)) \epsilon_{1} d \theta
\end{aligned}
$$

for all $t \geq T_{3}$. Then $I(t) \rightarrow-\infty$, as $t \rightarrow \infty$ by (129). This is a contradiction. Hence there is $\tau_{1} \geq T_{3}$ such that $I\left(\tau_{1}\right)<\epsilon_{1}$. Next, we will prove

$$
I(t) \leq \epsilon_{1}+a M w_{2} \epsilon_{0}
$$

for all $t \geq \tau_{1}$. Otherwise, there is $\tau_{2}>\tau_{1}$, such that $I\left(\tau_{2}\right)>$ $\epsilon_{1}+a M w_{2} \epsilon_{0}$. Hence, there must be $\tau_{3} \in\left(\tau_{1}, \tau_{2}\right)$ such that $I\left(\tau_{3}\right)=\epsilon_{1}$. Choose an integer $P \geq 0$ such that $\tau_{2} \in\left[\tau_{3}+\right.$ $\left.p w_{2}, \tau_{3}+(p+1) w_{2}\right]$. Integrating the second equation of system (122) from $\tau_{3}$ to $\tau_{2}$, we obtain

$$
\begin{aligned}
\epsilon_{1} & +a M w_{2} \epsilon_{0}<I\left(\tau_{2}\right)=I\left(\tau_{3}\right) \\
& +\int_{\tau_{3}}^{\tau_{2}}\left[\beta_{2}(\theta)\left(n_{2}(\theta)-I(\theta)-H(\theta)-R(\theta)\right) y\right. \\
& -(\mu(\theta)+d(\theta)) I(\theta)] d \theta \leq \epsilon_{1} \\
& +\int_{\tau_{3}}^{\tau_{2}}\left[\beta_{2}(\theta) M \varepsilon_{0}-(\mu(\theta)+d(\theta)) \epsilon_{1}\right] d \theta \leq \epsilon_{1} \\
& +\int_{\tau_{3}+p w_{2}}^{\tau_{2}}\left[\beta_{2}(\theta) M \varepsilon_{0}-(\mu(\theta)+d(\theta)) \epsilon_{1}\right] d \theta \\
& \leq \epsilon_{1}+a M w_{2} \epsilon_{0} .
\end{aligned}
$$

This is a contradiction. Hence, (135) is valid. 
If $R(t) \geq \epsilon_{3}$ for all $t \geq T_{3}$, then from the fourth equation of system (58) we have

$$
\begin{aligned}
R(t)-R\left(T_{3}\right) & =\int_{T_{3}}^{t}[\gamma(\theta) H(\theta)-\mu(\theta) R(\theta)] d \theta \\
& \leq \int_{T_{3}}^{t}\left[\gamma(\theta) \epsilon_{2}-\mu(\theta) \epsilon_{3}\right] d \theta
\end{aligned}
$$

for all $t \geq T_{3}$. By (130), it follows that $R(t) \rightarrow-\infty$ as $t \rightarrow$ $\infty$. This is a contradiction. Hence, there is $\tau_{1} \geq T_{3}$ such that $R\left(\tau_{1}\right)<\epsilon_{3}$. In the following, we prove

$$
R(t) \leq \epsilon_{3}+b w_{2} \epsilon_{3}
$$

for all $t \geq \tau_{1}$. If it is not true, then there is $\tau_{2}>\tau_{1}$ satisfying $R\left(\tau_{2}\right)>\epsilon_{3}+b w_{2} \epsilon_{3}$. Hence, there must be $\tau_{3} \in\left(\tau_{1}, \tau_{2}\right)$ such that $R\left(\tau_{3}\right)=\epsilon_{3}$ and $R(t)>\epsilon_{3}$ for all $t \in\left(\tau_{3}, \tau_{2}\right)$. Choose an integer $P \geq 0$ such that

$$
\tau_{2} \in\left[\tau_{3}+p w_{2}, \tau_{3}+(p+1) w_{2}\right] .
$$

Integrating the fourth equation of system (122) from $\tau_{3}$ to $\tau_{2}$, we obtain

$$
\begin{aligned}
\epsilon_{3}+b w_{2} \epsilon_{2}< & R\left(\tau_{2}\right) \\
= & R\left(\tau_{3}\right) \\
& \quad+\int_{\tau_{3}}^{\tau_{2}}[\gamma(\theta) H(\theta)-\mu(\theta) R(\theta)] d \theta \\
\leq & \epsilon_{3}+\int_{\tau_{3}}^{\tau_{2}}\left[\gamma(\theta) \epsilon_{2}-\mu(\theta) \epsilon_{1}\right] d \theta \\
\leq & \epsilon_{3}+\int_{\tau_{3}+p w_{2}}^{\tau_{2}} \gamma(\theta) \epsilon_{2} d \theta \leq \epsilon_{3}+b w_{2} \epsilon_{2} .
\end{aligned}
$$

This is a contradiction. Hence (138) is valid. From this we conclude that there exists $T_{0}>T_{3}$ such that (135) and (138) are both true for all $t \geq T_{0}$. For $t \geq 0$, we define a differentiable function

$$
\begin{aligned}
v(t) & =I(t) H(t), \\
v^{\prime}(t) & =I^{\prime} H+I H^{\prime} \\
= & {\left[\beta_{2}(t)\left(n_{2}(t)-I(t)-H(t)-R(t)\right) y(t)\right.} \\
- & (\mu(t)+d(t)) I(t)] H(t)+I(t) \\
\cdot & {\left[\beta_{3}(t)\left(n_{2}(t)-I(t)-H(t)-R(t)\right) H(t)\right.} \\
- & (\mu(t)+\alpha(t)+\gamma(t)) H(t)]=\beta_{2}(t)\left(n_{2}(t)\right. \\
- & I(t)-H(t)-R(t)) y(t) H(t)+\beta_{3}(t)\left(n_{2}(t)\right. \\
- & I(t)-H(t)-R(t)) I(t) H(t)-(\mu(t)+d(t) \\
+ & \mu(t)+\alpha(t)+\gamma(t)) H(t) \geq b\left(t, n_{2}(t)-I(t)\right. \\
- & H(t)-R(t)) v(t) \geq b\left(t, M-\epsilon_{1}-k \epsilon_{2}-\epsilon_{3}\right) \\
& \cdot v(t) .
\end{aligned}
$$

Integrating the above inequality from $T_{0}$ to $t$, we have

$$
v(t) \geq v\left(T_{0}\right) \exp \int_{T_{0}}^{t} b\left(s, M-\epsilon_{1}-k \epsilon_{2}-\epsilon_{3}\right) d s .
$$

By (131), we obtain $\lim \sup _{t \rightarrow \infty} v(t)=\infty$. This contradicts the boundedness of $I(t)$ and $H(t)$. From this, we have $\lim \sup _{t \rightarrow \infty} H(t)>\epsilon_{2}$. Secondly, we will prove that there is a constant $\nu_{1}>0$ such that

$$
\liminf _{t \rightarrow \infty} H(t) \geq v_{1} .
$$

From (129)-(132) and $\left(H_{2}\right)$, we have that there exist $T \geq T_{1}$, $P>0$, and $\eta>0$ such that

$$
\begin{gathered}
\int_{t}^{t+\alpha}\left[\beta_{2}(\theta) M \epsilon_{0}-(\mu(t)+d(t)) \epsilon_{1}\right] d \theta<-M, \\
\int_{t}^{t+\alpha}\left[\gamma(\theta) \epsilon_{2}-\mu(t) \epsilon_{3}\right] d \theta<-M, \\
\int_{t}^{t+\alpha} b\left(s, n_{2}(s)-\epsilon_{1}-k \epsilon_{2}-\epsilon_{3}\right) d s>\eta, \\
\int_{t}^{t+\alpha} \beta_{2}(\theta) d \theta>\eta
\end{gathered}
$$

for all $\alpha \geq P$ and $t \geq T$. Choose an integer $K_{0}>0$ such that

$$
e^{-(c+d) P} m v_{2} \eta e^{K_{0} \eta}>\epsilon_{1}+a M w_{2} \epsilon_{0},
$$

where $v_{2}=\epsilon_{2} e^{-(b+c) 2 P}$. By any $t_{0} \geq 0$, we claim that it is impossible that $H(t) \leq \epsilon_{2}$ for all $t \geq t_{0}$. From this claim, we will discuss the following two possibilities.

(i) $H(t) \geq \epsilon_{2}$ for all large $t$.

(ii) $H(t)$ oscillates about $\epsilon_{2}$ for all large $t$.

Finally, we will show that $H(t) \geq \epsilon_{2} e^{-(b+c)\left(K_{0}+2\right) P} \doteq v_{1}$ as $t$ is large sufficiently. Let $t_{1}$ and $t_{2}$ be sufficiently large time satisfying

$$
\begin{aligned}
H\left(t_{1}\right) & =H\left(t_{2}\right)=\epsilon_{2}, \\
H(t) & <\epsilon_{2}
\end{aligned}
$$

for all $t \in\left(t_{1}, t_{2}\right)$.

$$
\text { If } t_{2}-t_{1} \leq\left(K_{0}+2\right) P \text {, then } H^{\prime}(t)=\beta_{3}(t)\left(n_{2}(t)-I(t)-\right.
$$
$H(t)-R(t)) H(t)-(\mu(t)+\alpha(t)+\gamma(t)) H(t) \geq-(b+c+e) H(t)$. And $H\left(t_{1}\right)=\epsilon_{2}$, and we have $H(t) \geq \epsilon_{2} e^{-(b+c)\left(K_{0}+2\right) P}$ for all $t \in\left[t_{1}, t_{2}\right]$. If $t_{2}-t_{1}>\left(K_{0}+2\right) P$, then it is clear that $H(t) \geq$ $\epsilon_{2} e^{-(b+c)\left(K_{0}+2\right) P}$ for all $t \in\left[t_{1}, t_{1}+\left(K_{0}+2\right) P\right]$. If $I(t) \geq \epsilon_{1}$ for all $t \in\left[t_{1}, t_{1}+P\right]$, then

$$
\begin{aligned}
& I\left(t_{1}+P\right)=I\left(t_{1}\right)+\int_{t_{1}}^{t_{1}+P}\left[\beta_{2}(\theta) S(\theta) y(\theta)\right. \\
& \quad-(\mu(t)+d(t)) I(\theta)] d \theta \leq M \\
& \quad+\int_{t_{1}}^{t_{1}+P}\left[\beta_{2}(\theta) M \epsilon_{1}-(\mu(\theta)+d(\theta)) \epsilon_{2}\right] d \theta \\
& \quad<0 .
\end{aligned}
$$


This is a contradiction. Hence, there is $\bar{t} \in\left[t_{1}, t_{1}+P\right]$ such that $I(\bar{t})<\epsilon_{1}$.

From (135), we can obtain

$$
I(t) \leq \epsilon_{1}+a M w_{2} \epsilon_{0}
$$

for all $t \in\left[\bar{t}, t_{2}\right]$. Similarly, there is $\tilde{t} \in\left[t_{1}, t_{1}+P\right]$, such that $R(\widetilde{t})<\epsilon_{3}$ and

$$
R(t) \leq \epsilon_{3}+b w_{2} \epsilon_{2}
$$

for all $t \in\left[\widetilde{t}, t_{2}\right]$. Obviously, as $t \in\left[t_{1}, t_{1}+2 P\right]$,

$$
H(t) \geq \epsilon_{2} e^{-(b+c+e) 2 P} \doteq v_{2}>v_{1} .
$$

Therefore, from the second equation of system (122), (132), (151), and (152), we have

$$
\begin{aligned}
I^{\prime}(t)= & \beta_{2}(t)\left(n_{2}(t)-I(t)-H(t)-R(t)\right) y(t) \\
& -(\mu(t)+d(t)) I(t) \\
\geq & \beta_{2}(t)\left(n_{2}(t)-\epsilon_{1}-k \epsilon_{2}-\epsilon_{3}\right) y(t) \\
& -(\mu(t)+d(t)) I(t) \\
\geq & m v_{2} \beta_{2}(t)-(c+d) I(t) .
\end{aligned}
$$

For all $t \in\left[t_{1}+P, t_{1}+2 P\right]$, integrating the above inequality from $t_{1}+P$ to $t_{1}+2 P$ and using (147) we have

$$
\begin{aligned}
& I\left(t_{1}+2 P\right) \geq e^{-(c+d)\left(t_{1}+2 P\right)}\left[I\left(t_{1}+P\right) e^{-(c+d)\left(t_{1}+P\right)}\right. \\
& \left.\quad+\int_{t_{1}+P}^{t_{1}+2 P} m v_{2} \beta_{2}(u) e^{(c+d) u}\right] d u \\
& \quad \geq e^{-(c+d)\left(t_{1}+2 P\right)} \int_{t_{1}+P}^{t_{1}+2 P} m v_{2} \beta_{2}(u) e^{(c+d) u} d u \\
& \quad \geq e^{-(c+d) P} m v_{2} \int_{t_{1}+P}^{t_{1}+2 P} \beta_{2}(u) d u>e^{-(c+d) P} m v_{2} \eta .
\end{aligned}
$$

We claim that $H(t) \geq v_{1}$ for all $t \in\left[t_{1}+\left(K_{0}+2\right) P, t_{2}\right]$. If it is not true, then there is $T_{0} \geq 0$ such that $H\left(t_{1}+\left(K_{0}+2\right) P+T_{0}\right)=v_{1}$ and $H(t) \geq v_{1}$ on $\left[t_{1}, t_{1}+\left(K_{0}+2\right) P+T_{0}\right]$. Let $v(t)=I(t)+H(t)$, and $t_{0}=t_{1}+\left(K_{0}+2\right) P+T_{0}$, and the derivative of $v(t)$ along solution of (122) satisfies

$$
v^{\prime}(t) \geq b\left(t, M-\epsilon_{1}-k \epsilon_{2}-\epsilon_{3}\right) v(t)
$$

for all $t \in\left[t_{1}+2 P, t_{1}\right]$. Integrating the above inequality from $t_{1}+2 P$ to $t_{0}$, we further have

$$
\begin{aligned}
& I\left(t_{0}\right)+v_{1} \geq\left[I\left(t_{1}+2 P\right)+H\left(t_{1}+2 P\right)\right] \\
& \cdot \exp \left(\int_{t_{1}+2 P}^{t_{0}} b\left(t, M-\epsilon_{1}-k \epsilon_{2}-\epsilon_{3}\right) d t\right) \\
& \quad \geq e^{-(c+d) P} m v_{1} v_{2} \eta e^{K_{0} \eta}>\left(\epsilon_{1}+a M w_{2} \epsilon_{0}\right) v_{1} \\
& \quad \geq\left(e^{-(c+d) P} m v_{2} \eta+v_{1}\right) e^{K_{0} \eta} \geq\left(\epsilon_{1}+a M w_{2} \epsilon_{0}\right) \\
& +v_{1} e^{K_{0} \eta} .
\end{aligned}
$$

This contradicts (151). Hence $H(t) \geq \nu_{1}$ is valid for any $t \in$ $\left[t_{1}, t_{2}\right]$ and we have

$$
\liminf _{t \rightarrow \infty} H(t) \geq v_{1}>0
$$

Theorem 14. Suppose that assumptions $\left(H_{1}\right)$ and $\left(H_{2}\right)$ hold. If there is a constant $\lambda^{\prime \prime}>0$, such that

$$
\begin{array}{r}
R_{1}^{*}=\limsup _{t \rightarrow \infty} \int_{t}^{t+\lambda^{\prime \prime}}\left[\beta_{3}(\theta) n_{2}(\theta)-\mu(t)\right] d \theta \leq 0, \\
\text { or } R_{2}^{*}=\limsup _{t \rightarrow \infty} \frac{1}{t} \int_{0}^{t}\left[\beta_{3}(\theta) n_{2}(\theta)-\mu(t)\right] d \theta<0,
\end{array}
$$

then infective $H$ in system (122) is extinct.

Proof. From assumption $\left(\mathrm{H}_{2}\right)$ we choose $\eta>0$ small enough and $T_{1}>0$ big enough, satisfying

$$
\int_{t}^{t+w_{2}} \beta_{3}(\theta) d \theta \geq \eta
$$

for all $t \geq T_{1}$. For any constant $0<\epsilon<1$, we set

$$
\epsilon_{0}=\min \left\{\frac{\lambda^{\prime \prime} \eta \epsilon}{2 w_{2}}, \frac{1}{2} \eta \epsilon\right\}>0 .
$$

If (159) holds, then there exists $T_{2} \geq T_{1}$ such that

$$
\int_{t}^{t+\lambda^{\prime \prime}}\left[\beta_{3}(\theta) n_{2}(\theta)-\mu(t)\right] d \theta \leq \epsilon_{0}
$$

for all $t \geq T_{2}$. Choose an integer $n^{\prime}$ satisfying $2 w_{2} / \lambda^{\prime \prime} \leq n^{\prime} \leq$ $2 w_{2} / \lambda^{\prime \prime}+1$. Set $\lambda_{0}=n^{\prime} \lambda^{\prime \prime}$, and then

$$
\begin{gathered}
\int_{t}^{t+\lambda_{0}}\left[\beta_{3}(\theta) n_{2}(\theta)-\mu(\theta)-\beta_{3}(\theta) \epsilon\right] d \theta \\
\leq \int_{t}^{t+n^{\prime} \lambda^{\prime \prime}}\left[\beta_{3}(\theta) n_{2}(\theta)-\mu(\theta)\right] d \theta \\
\quad-\int_{t}^{t+2 w_{2}} \beta_{3}(\theta) \epsilon d \theta \leq n^{\prime} \epsilon_{0}-2 \eta \epsilon .
\end{gathered}
$$

Then $n^{\prime} \epsilon_{0}-2 \eta \epsilon \leq-(1 / 2) \eta \epsilon \doteq-\eta^{*}<0$. Set function

$$
v(t)=R(t)+H(t),
$$

and differentiate $v$ along a solution of (122) obtaining

$$
\begin{aligned}
& v^{\prime}(t)=R^{\prime}+H^{\prime}=\gamma(t) H(t)-\mu(t) R(t)+\beta_{3}(t) \\
& \cdot\left(n_{2}(t)-I(t)-H(t)-R(t)\right) H(t)-(\mu(t) \\
& +\alpha(t)+\gamma(t)) H(t)=\beta_{3}(t)\left(n_{2}(t)-I(t)-H(t)\right. \\
& -R(t)) H(t)-\mu(t) R(t)-(\mu(t)+\alpha(t)+\gamma(t)) \\
& \cdot H(t) \leq \beta_{3}(t)\left(n_{2}(t)-I(t)-H(t)-R(t)\right) v(t) \\
& -\mu(t) v(t)=\left[\beta_{3}(t) n_{2}(t)\right. \\
& \left.-\beta_{3}(t)(I(t)+H(t)+R(t))-\mu(t)\right] v(t) \\
& \leq\left[\beta_{3}(t) n_{2}(t)-\beta_{3}(t) v(t)-\mu(t)\right] v(t) .
\end{aligned}
$$


If $v(t) \geq \epsilon$ for all $t \geq T_{2}$, then from (166) we obtain

$$
\begin{aligned}
& v(t) \\
& \quad<v\left(T_{2}\right) \exp \int_{T_{2}}^{t}\left[\beta_{3}(s) n_{2}(s)-\beta_{3}(s) \epsilon-\mu(s)\right] d s .
\end{aligned}
$$

By (164) and (167), it follows that $v(t) \rightarrow 0$ as $t \rightarrow \infty$. This is a contradiction with $v(t) \geq \epsilon$. Hence, there must be $t_{1} \geq T_{2}$ such that $v\left(t_{1}\right)<\epsilon_{1}$. Let

$$
n(\epsilon)^{\prime}=\sup _{t \geq T_{2}}\left|\beta_{3}(t) n_{2}(t)-\mu(t)\right|+\beta_{3}(t) \epsilon
$$

be bounded for each $\epsilon \in(0,1)$. Finally, we will prove

$$
v(t) \leq \epsilon \exp \left(n(\epsilon)^{\prime} \lambda_{0}\right)
$$

for all $t \geq t_{1}$. If it is not true, then there exists $t_{2}>t_{1}$, such that

$$
v\left(t_{2}\right)>\epsilon \exp \left(n(\epsilon)^{\prime} \lambda_{0}\right)
$$

Hence, there exists $t_{3} \in\left(t_{1}, t_{2}\right)$ such that $v\left(t_{3}\right)=\epsilon$ and $v(t)>\epsilon$ for all $t \in\left(t_{3}, t_{2}\right)$. Let $P$ be a nonnegative integer such that $t_{2} \in\left(t_{3}+P \lambda_{0}, t_{3}+(P+1) \lambda_{0}\right)$. From (167), we have

$$
\begin{aligned}
& \epsilon \exp \left(n(\epsilon)^{\prime} \lambda_{0}\right)<v\left(t_{2}\right) \\
& \quad \leq v\left(t_{3}\right) \exp \int_{t_{3}}^{t_{2}}\left[\beta_{3}(t) n_{2}(t)-\beta_{3}(t) \epsilon-\mu(t)\right] d t \\
& \quad \leq \epsilon \exp \left(n(\epsilon)^{\prime} \lambda_{0}\right) .
\end{aligned}
$$

This leads to a contradiction. Hence, inequality (169) holds. Furthermore, since $\epsilon$ can be arbitrarily small and $0 \leq H(t) \leq$ $v(t)$ and $0 \leq I(t) \leq v(t)$, we conclude that $I(t) \rightarrow 0$ and $H(t) \rightarrow 0$ as $t \rightarrow \infty$. Suppose that (160) holds. There exist $\delta>0$ and $T_{0}>0$ such that

$$
\frac{1}{t} \int_{0}^{t}\left[\beta_{3}(\theta) n_{2}(\theta)-\mu(\theta)\right] d \theta<-\delta
$$

for all $t \geq T_{0}$. From (167) we directly obtain

$$
v(t) \leq v\left(T_{0}\right) \exp \int_{T_{2}}^{t}\left[\beta_{3}(s) n_{2}(s)-\mu(s)\right] d s
$$

for all $t \geq T_{0}$. By (172), $v(t) \rightarrow 0$ as $t \rightarrow \infty$. Therefore, we finally also have $I(t) \rightarrow 0$ and $H(t) \rightarrow 0$ as $t \rightarrow \infty$. This completes the proof of Theorem 14.

\section{Concluding Remarks}

Birds and poultry are the source of food and livelihoods in many parts of the world where avian influenza is endemic. The avian influenza virus is mainly transmitted directly from birds or from avian virus-contaminated environments to humans. However, at least one instance of the human-tohuman spread is thought to have occurred in Thailand. We assume that avian influenza can be transmitted between people. Based on the reported data and experiments, we construct the nonautonomous avian influenza model which incorporates the effect of climate change as parameters become time dependent. These models are more reasonable and closely match with the realistic situation. When there is no infected individual with mutant avian influenza (i.e., $H=$ 0 ), system (13) is ultimately bounded and the disease-free equilibrium is globally stable if $r_{0}^{\max }<1$. If $r_{0}^{\min }>1$, system (13) is uniformly persistent. When mutant avian influenza occurs in human, that is, when $(H \neq 0), E_{0}^{\prime}$ is globally stable when $R_{0}^{\max }<1$. In human-poultry system, we have given the conditions for the permanence of the system; that is, if $\left(H_{1}\right)$ and $\left(H_{2}\right)$ hold, $y(t)$ is permanent and strongly persistent. Thus we have $R_{0}^{*}>0$. If $R_{1}^{*} \leq 0$ or $R_{2}^{*}<0$, the infective $y$ is extinct. In human system, if $\lambda>0$ and $R_{0}^{*}>0$, the infective $H(t)$ is permanent under the condition of $\left(H_{1}\right)$ and $\left(H_{2}\right)$. If there is a constant $\lambda>0$ such that $R_{1}^{*} \leq 0$ or $R_{2}^{*}<0$, the infective $H(t)$ is extinct.

\section{Competing Interests}

The authors declare that there is no conflict of interests.

\section{Acknowledgments}

This research is supported by the NNSF of China (no. 11271314), Plan for Scientific Innovation Talent of Henan Province (144200510021), and Plan for Research Projects of Shaoguan University (no. S201501014).

\section{References}

[1] World Health Organization, Avian Influenza Fact Sheet, World Health Organization, Geneva, Switzerland, 2014, http://www .who.int/mediacentre/factsheets/avian_influenza/en/.

[2] Z. Teng, Y. Liu, and L. Zhang, "Persistence and extinction of disease in non-autonomous SIRS epidemic models with diseaseinduced mortality," Nonlinear Analysis: Theory, Methods \& Applications, vol. 69, no. 8, pp. 2599-2614, 2008.

[3] T. Zhang and Z. Teng, "On a nonautonomous SEIRS model in epidemiology," Bulletin of Mathematical Biology, vol. 69, no. 8, pp. 2537-2559, 2007.

[4] N. Bacaer and S. Guernaoui, "The epidemic threshold of vector-borne diseases with seasonality. The case of cutaneous leishmaniasis in Chichaoua, Morocco," Journal of Mathematical Biology, vol. 53, no. 3, pp. 421-436, 2006.

[5] J. Ma and Z. Ma, "Epidemic threshold conditions for seasonally forced SEIR models," Mathematical Biosciences and Engineering, vol. 3, no. 1, pp. 161-172, 2006.

[6] F. A. Coutinho, M. N. Burattini, L. F. Lopez, and E. Massad, "Threshold conditions for a non-autonomous epidemic system describing the population dynamics of dengue," Bulletin of Mathematical Biology, vol. 68, no. 8, pp. 2263-2282, 2006.

[7] H. R. Thieme, "Uniform persistence and permanence for nonautonomous semiflows in population biology," Mathematical Biosciences, vol. 166, no. 2, pp. 173-201, 2000.

[8] T. Zhang, J. Liu, and Z. Teng, "Differential susceptibility timedependent SIR epidemic model," International Journal of Biomathematics, vol. 1, no. 1, pp. 45-64, 2008. 
[9] X. Abdurahman, "On the persistence and extinction for a nonautonomous SIRS epidemic model," Journal of Biomathematics, vol. 21, no. 2, pp. 167-176, 2006.

[10] K. Wang and A. Fan, "Uniform persistence and periodic solution of chemostat-type model with antibiotic," Discrete and Continuous Dynamical Systems, vol. 4, no. 3, pp. 789-795, 2004.

[11] S. Iwami, Y. Takeuchi, and X. Liu, "Avian-human influenza epidemic model," Mathematical Biosciences, vol. 207, no. 1, pp. 1-25, 2007.

[12] S. Iwami, Y. Takeuchi, A. Korobeinikov, and X. Liu, "Prevention of avian influenza epidemic: what policy should we choose?" Journal of Theoretical Biology, vol. 252, no. 4, pp. 732-741, 2008.

[13] S. Iwami, Y. Takeuchi, and X. Liu, "Avian flu pandemic: can we prevent it?" Journal of Theoretical Biology, vol. 257, no. 1, pp. 181190, 2009.

[14] S. Iwami, Y. Takeuchi, X. Liu, and S. Nakaoka, "A geographical spread of vaccine-resistance in avian influenza epidemics," Journal of Theoretical Biology, vol. 259, no. 2, pp. 219-228, 2009.

[15] J.-S. Kwon, H.-J. Lee, D.-H. Lee et al., "Immune responses and pathogenesis in immunocompromised chickens in response to infection with the H9N2 low pathogenic avian influenza virus," Virus Research, vol. 133, no. 2, pp. 187-194, 2008.

[16] H. Wang, Z. Feng, Y. Shu et al., "Probable limited personto-person transmission of highly pathogenic avian influenza A(H5N1) virus in China," The Lancet, vol. 371, no. 9622, pp. 1427-1434, 2008.

[17] L. Bourouiba, A. Teslya, and J. Wu, "Highly pathogenic avian influenza outbreak mitigated by seasonal low pathogenic strains: insights from dynamic modeling," Journal of Theoretical Biology, vol. 271, pp. 181-201, 2011.

[18] T. M. Uyeki and N. J. Cox, "Global concerns regarding novel influenza A (H7N9) virus infections," The New England Journal of Medicine, vol. 368, no. 20, pp. 1862-1864, 2013.

[19] Y. Chen and Y. Wen, "Global dynamic analysis of a H7N9 avian-human influenza model in an outbreak region," Journal of Theoretical Biology, vol. 367, pp. 180-188, 2015.

[20] F. Li and A. Qi, "Mathematical model of the effects of plasma cortisol on human T lymphocytes recirculation," Acta Biochimica et Biophysica Sinica, vol. 16, pp. 586-594, 2000. 


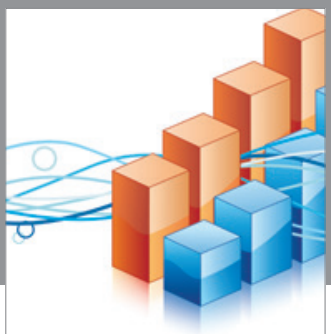

Advances in

Operations Research

vatem alat4

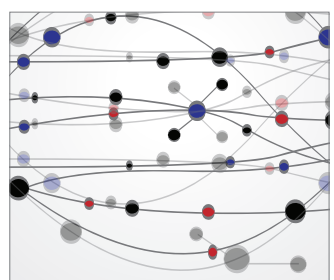

\section{The Scientific} World Journal
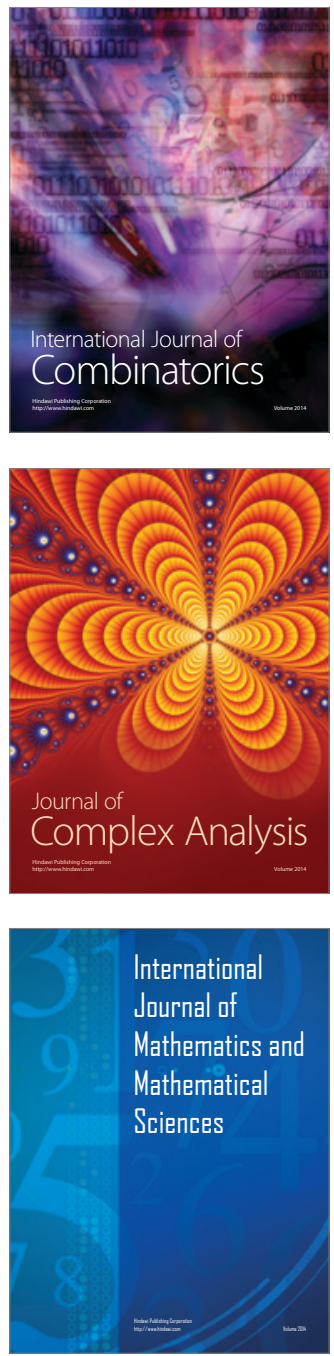
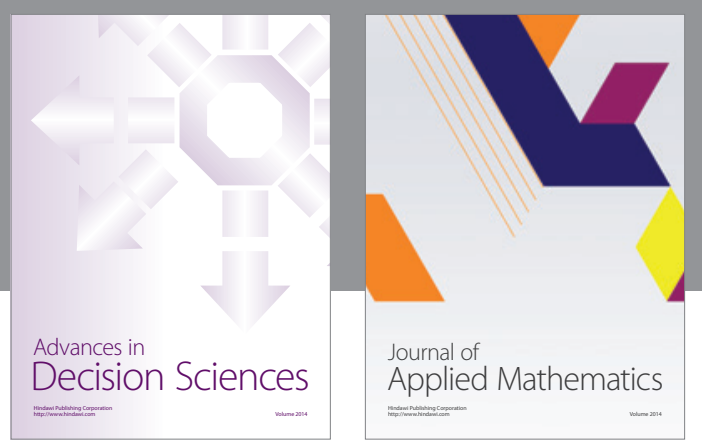

Algebra

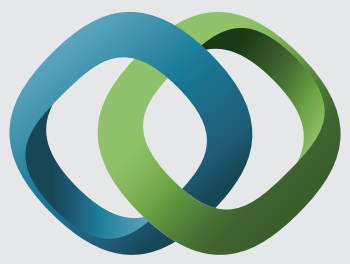

\section{Hindawi}

Submit your manuscripts at

http://www.hindawi.com
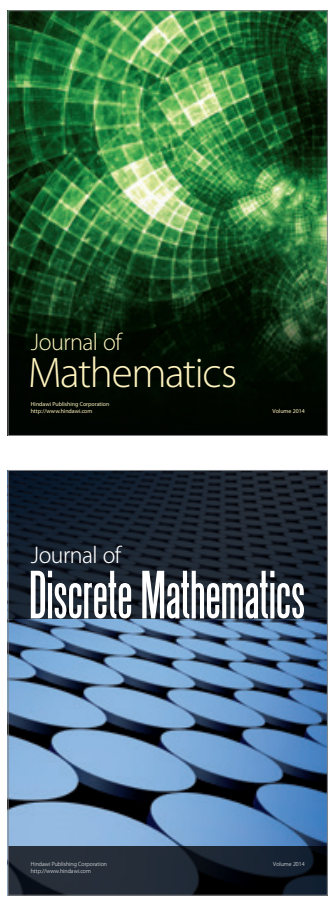

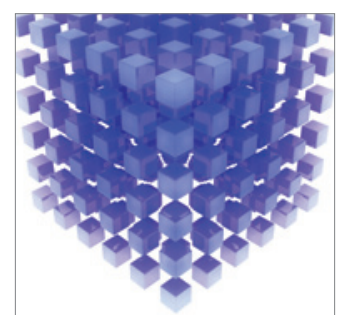

Mathematical Problems in Engineering
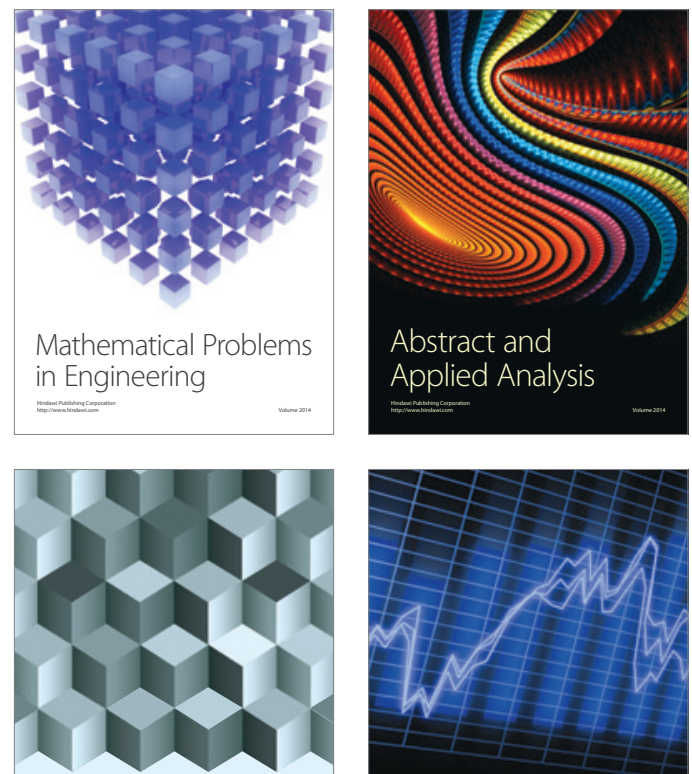

Journal of

Function Spaces

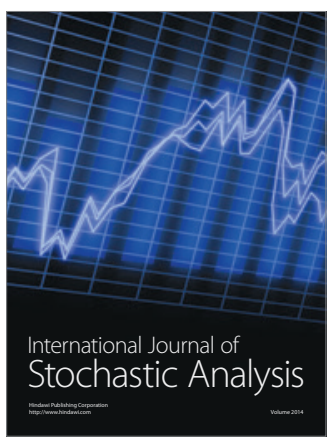

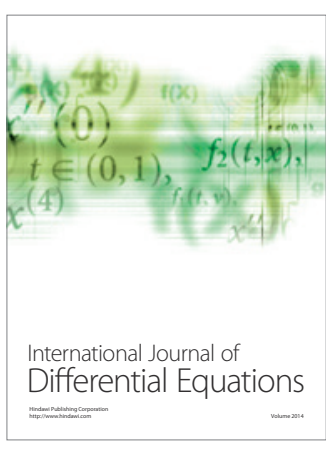
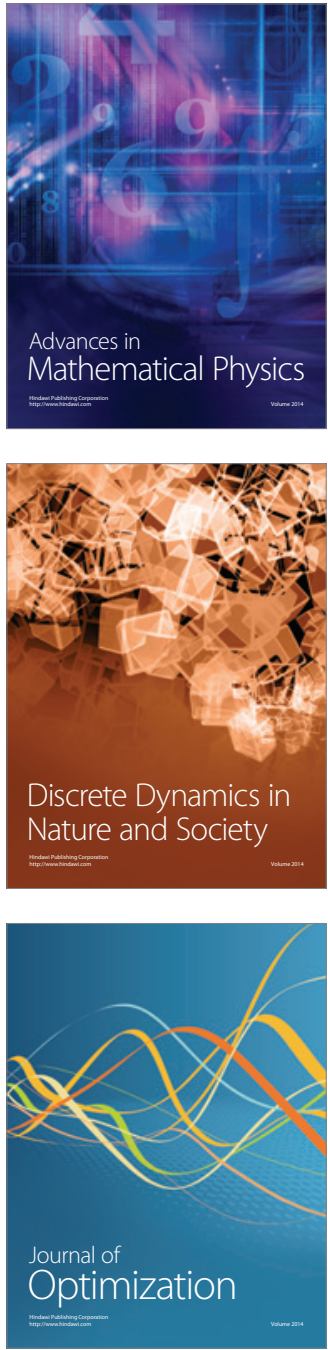